\title{
Growth of Transition Metal Dichalcogenides and Directly Modulating Their Properties by Chemical Vapor Deposition
}

\author{
Lei Tong, ${ }^{\dagger, a}$ Tiande Liu, ${ }^{\dagger, a}$ Renli Liang, ${ }^{b}$ ShuaiWang, ${ }^{b}$ Jingwen Chen, ${ }^{b}$ Jiangnan Dai, ${ }^{,, b}$ and Lei Ye ${ }^{*, a}$ \\ ${ }^{a}$ School of Optical and Electronic Information, Huazhong University of Science and Technology, \\ Wuhan, Hubei 430074, China \\ ${ }^{b}$ Wuhan National Laboratory for Optoelectronics, Huazhong University of Science and Technology, \\ Wuhan, Hubei 430074, China
}

\begin{abstract}
The layered structure of transition metal dichalcogenides (TMDs) gives rise to many novel properties for functional applications in a wide range of fields. However, successful synthesis of TMDs and directly modulating properties of TMDs during the growth process are facing great challenges, which limits their future practical applications. In this review, we focus on current state of the art chemical vapor deposition (CVD) synthesis of TMDs alloys, convenient methods to modulate properties of TMDs by CVD. Then, TMDs-based lateral and vertical heterostructures utilizing CVD methods are reviewed. Finally, we summarize patterned growth of TMDs briefly.
\end{abstract}

Keywords transition metal dichalcogenides, chemical vapor deposition, doping and alloying, heterostructure growth, pattern growth

\section{Introduction}

Since the discovery of graphene, two dimensional materials have attracted much attention. Among diverse two-dimensional materials, the most studies are transition metal dichalcogenides (TMDs) with intra-layer atoms structure which is covalent bonded, while interlayer atoms are held together through van del Waals interactions. ${ }^{[1]}$ TMDs have diverse bandgap from semiconductors, semimetals to metals characters, and the bandgap can be modulated by reducing their thickness, ${ }^{[2]}$ applying strain or pressure ${ }^{[3-5]}$ doping and alloying, introducing grain boundaries or defects. ${ }^{[6,7]}$ Type II band alignment can be achieved easily by TMDs-based vertical heterostructures to harvest low energy photons. ${ }^{[8,9]}$ Due to the layered nature of TMDs, electron-photon, electron-electron, electron-phonon interactions are much stronger within confined two-dimensional planes, providing superior characters than traditional bulk materials. ${ }^{[10-13]}$ TMDs are considered as the promising materials applying in the fields of electronics, optoelectronics, Weyl semimetals. ${ }^{[14-16]}$ Considering the elasticity nature of ultra-thin TMDs, they are also compatible with flexible electronics. ${ }^{[3,17]}$ In addition, for most TMDs, the inversion symmetry is naturally broken as a result of odd layer numbers or distorted crystal structure, combined with strong spin-orbit coupling within the layers, to give rise to valley-contrasting optical selection rule, leading to TMDs as ideal materials for valley- and spin- electronics. ${ }^{[18-29]}$ The unique orbital hybrid structure between transition metal atoms and chalcogenide atoms endows TMDs with special properties under quantum states or ultra-low temperature conditions, superconductivity, ${ }^{[24,30-32]}$ topological insulators, ${ }^{[33-36]}$ nonsaturated magnetoresistance, ${ }^{[23,37-41]}$ ferromagnetism and anti-ferromagnetism, ${ }^{[42]}$ have trigged many research thrust. Moreover, TMDs are compatible with current fabrication technologies, integration with waveguides, resonance cavities to utilize nonlinear optical phenomenon or output nanolaser, or integration with meta-surfaces, plasmon to realize special function are very promising based on TMDs. ${ }^{[43-48]}$

However, most applications mentioned above are based on exfo- liated samples, limited by the low yields, relative small physical size and uncontrollable layer numbers, complicated transfer methods for device fabrication, obstructing their future practical applications. Thus, the bottleneck remains the need for reliable synthesis methods for TMDs crystals with large size and high quality. Many efforts have been paid to synthesize high quality large size uniform TMDs layers using chemical vapor deposition (CVD) methods in recent years, but many methods are still in the infancy, irregular flake edges, grain boundary and atom defects limit device performances. These problems limit crystal size and hinder integration into complicated device systems. To control band structure of TMDs effectively, doping and alloying based on CVD are most investigated methods. But lattice mismatch between different components will lead to non-uniform strain on the flake, resulting in more defects or non-uniformly distributed dopants. Therefore, these methods are also facing great challenges. In addition, free from dangling bonds makes TMDs ideal materials for heterostructures, but contaminations during transfer procedure or staking order mismatch will degrade device performances seriously. Lateral heterostructures with sharp interface is hard to be fabricated through transfer. CVD based heterostructure synthesis is another challenge in the field. To further promote the development of TMDs-based applications, investigations into CVD synthesis methods are of significant importance.

In this review, we focus on CVD growth of various TMDs. We introduce state of the art doping and alloying achievements. Then, we introduce lateral and vertical heterostructure synthesis of multicomponent TMDs. Such CVD grown heterostructures are promising in a wide range of fields. Finally, we introduce CVD growth of patterned TMDs briefly. Those researches give an insight into future research hotspots, which focus not only on the growth methods, functional structure, but also on the physical and thermodynamic mechanisms of TMDs deposition, controllability of atom scale crystal structures such as vacancies and grain boundaries. A brief summary and prospects for future researches are also provided in the last part.

\footnotetext{
* E-mail: daijiangnan@mail.hust.edu.cn, leiye@mail.hust.edu.cn. ${ }^{\dagger}$ These authors contributed equally. Received September 12, 2017; accepted November 3, 2017.
}

Gen. Chem. 2017, 3, 182-193 


\section{Alloying synthesis}

Alloying is a general strategy to turn carrier types and carrier densities of materials, ${ }^{[49-55]}$ which is more convenient than common methods such as electrostatic gating and strain modulation. Remarkably, alloying is effective to modulate band structure of materials to realize a continuous broader band response for nanoelectronics and nanophotonics. ${ }^{[52,54-59]}$ Optical properties and superconductivity can also be modulated by these methods. ${ }^{[14,60]}$ Besides, alloying is efficient to improve stability in ambient conditions. ${ }^{[55,61]}$ However, due to the challenges in controlling uniform dopant distribution, many works are still under investigation. In this part, we introduce current state of the art alloying achievements based on CVD and chemical vapor transport (CVT) methods.

\section{$\mathrm{MoS}_{2}$ alloying}

$\mathrm{MoS}_{2}$ is the most studied material at present. But monolayer $\mathrm{MoS}_{2}$ possesses large bandgap of $1.86 \mathrm{eV}$, which limits its applications in the visible range. To modulate properties of $\mathrm{MoS}_{2}$, the widely used dopant is Se atom. Duan et al. have proposed a CVD method to synthesize $\mathrm{MoS}_{2(1-x)} \mathrm{Se}_{2 x}$ flakes (Figure 1). ${ }^{[56]} \mathrm{S}$ and Se powder are placed on the upstream position separately. $\mathrm{MoO}_{3}$ powder is on the heating zone. The composition of product changes in different positions of silicon wafer. S content decreases gradually when the flake is away from furnace center, which is relevant to vapor distribution in the growth procedure. ${ }^{[56]}$ Photoluminescence measurements reveal that the emission wavelength changes continuously from $668 \mathrm{~nm}$ $\left(\mathrm{MoS}_{2}\right)$ to $795 \mathrm{~nm}\left(\mathrm{MoSe}_{2}\right)$. The product shows p-type to n-type transfer with increased $\mathrm{S}$ content, when $x$ is between 0.4 and 0.65 . It shows ambipolar characters. ${ }^{[56]}$ Different from complex CVD methods, Xie et al. have realized $\mathrm{MoS}_{2(1-x)} \mathrm{Se}_{2 x}$ monolayer growth using low-pressure physical vapor deposition (PVD) method in a threezone furnace by controlling evaporation temperature, deposition temperature and $\mathrm{H}_{2}$ flow rate. ${ }^{[57]}$ The amount of both sources is the key parameter to grow triangle flakes or films. The bandgap can be narrowed from 1.86 to $1.73 \mathrm{eV}$ with increased Se contents. ${ }^{[57,58]} \mathrm{Re}$ markably, polarized Raman spectra are efficient to detect properties of the product, while pure $\mathrm{MoS}_{2}$ is isotropic. FET test reveals that $\mathrm{MoS}_{2(1-x)} \mathrm{Se}_{2 x}$ monolayer shows n-type transport behavior with high on-off ratio larger than $10^{5}$, showing that the alloy characters are not degraded comparing with pure $\mathrm{MoS}_{2 .}{ }^{[57,62,63]}$ Ajayan et al. also achieved synthesis of $\mathrm{MoS}_{2(1-x)} \mathrm{Se}_{2 x}$ alloy, Se component can be controlled from 0 to $75 \%$ by changing ratio of $\mathrm{S}$ and Se precursors before growth procedure, and prolonged growing time could result to film growth due to increased nucleation density. ${ }^{[64]}$ However, the optical bandgap of the alloy can be turned over $200 \mathrm{meV}$. Except for common Raman and PL measurements, X-ray photoelectron spectroscopy (XPS) is valid to confirm Se content by comparing Se 3p3/2 and 3d peak intensity revolution, while atomic annular dark field (ADF) imaging is useful to analyze dopant distribution. ${ }^{[64]}$ Different from doping in the growth process, atom substitution can be conducted after growth of $\mathrm{MoS}_{2}$, and annealing using the dopant sources in the furnace is also efficient to turn band structure of the material. ${ }^{[65]}$

Except for chalcogenide atom substitution, transition metal atoms can also be substituted using CVT methods to modulate properties of $\mathrm{MoS}_{2}$ and these alloys shows good thermodynamic stability at ambient conditions. ${ }^{[55,66]}$ However, transition metal atoms are harder to be replaced using CVD methods because they are more stable in the crystal structure and particular electrical and optoelectronic properties may be degraded in CVD grown alloys. ${ }^{[67,68]} \mathrm{W}$ doped $\mathrm{MoS}_{2}$ can be synthesized through CVT methods, and the bandgap can be tuned with changed $\mathrm{W}$ contents. ${ }^{[54,55]}$ Robinson et al. realized manganese doping in monolayer $\mathrm{MoS}_{2}$ (Figure 2). ${ }^{[68]}$ Interestingly, the alloying of Mo atoms is only achievable on inert substrate. However, the PL of the alloy is quenched, which limits future applications based on this alloy. ${ }^{[68]} \mathrm{Nb}$-doped $\mathrm{MoS}_{2}$ with a degenerate hole density can be realized through CVT method. ${ }^{[69]}$

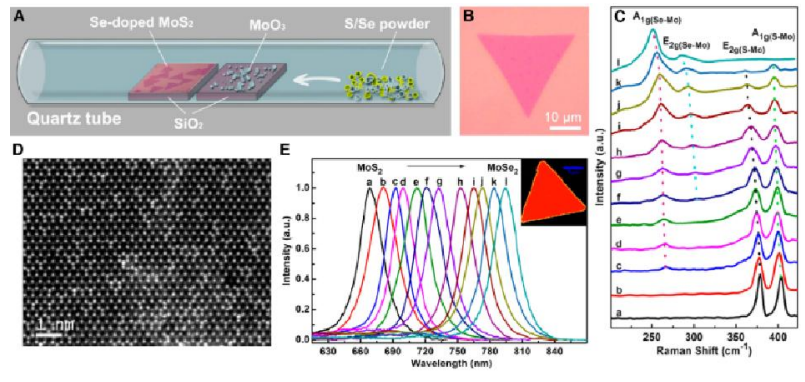

Figure 1 CVD grown $\operatorname{MoS}_{2(1-x)} \mathrm{Se}_{2 x}$ alloy. (A) Schematic of CVD synthesis of $\mathrm{MoS}_{2(1-x)} \mathrm{Se}_{2 x}$ layers. (B) Optical image of $\mathrm{MoS}_{2(1-x)} \mathrm{Se}_{2 x}$ flake. (C) Raman spectra of the $\mathrm{MoS}_{2(1-x)} \mathrm{Se}_{2 x}$ layers with increased Se contents from a to 1. (D) ADF image of Se-doped $\mathrm{MoS}_{2}$. (E) PL spectra of the $\mathrm{MoS}_{2(1-x)} \mathrm{Se}_{2 x}$ layers with increased Se contents from a to 1 . (A), (B) and (D) are reproduced with permission from Ref. [64]. (C) and (E) are reproduced with permission from Ref. [56].
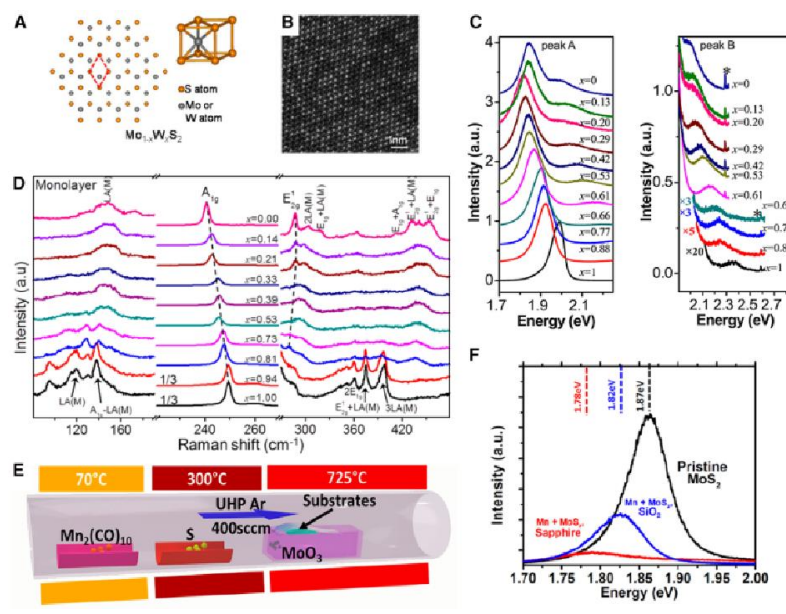

Figure 2 Transition metal alloying of $\mathrm{MoS}_{2}$. (A) Crystal structure of $\mathrm{Mo}_{1-x} \mathrm{~W}_{x} \mathrm{~S}_{2}$ alloy. (B) Scanning tunnel electron microscope (STEM) image of $\mathrm{Mo}_{0.47} \mathrm{~W}_{0.53} \mathrm{~S}_{2}$ alloy monolayer. (C) PL spectra of $\mathrm{Mo}_{1-x} \mathrm{~W}_{x} \mathrm{~S}_{2}$ alloy with different $\mathrm{W}$ contents, the left figure is the A peak, the right figure is the B peak. (D) Raman spectra of $\mathrm{Mo}_{1-x} \mathrm{~W}_{x} \mathrm{~S}_{2}$ alloy with different $\mathrm{W}$ contents. (E) Schematic of CVD growth of Mn alloyed $\mathrm{MoS}_{2}$. (F) PL quenching of Mn alloyed $\mathrm{MoS}_{2}$ on $\mathrm{SiO}_{2}$ and sapphire substrate. (A) (B) (C) are reproduced with permission from Ref. [55]. (D) is reproduced with permission from Ref. [54]. (E) and (F) are reproduced with permission from Ref. [68].

\section{MoTe $_{2}$ alloying}

In recent years, $\mathrm{MoTe}_{2}$ has attracted much attention due to multiphase transition, ${ }^{[70-73]}$ Weyl semimetal, ${ }^{[30]}$ giant negative magnetoresistance and superconductivity. Doping is an efficient way to turn those properties. $^{[14]}$

Chen et al. have realized $\mathrm{Mo}_{1-x} \mathrm{~W}_{x} \mathrm{Te}_{2}$ CVT growth by solid state reaction at $1073 \mathrm{~K}$ utilizing a mixture of high purity elemental powders W, Mo and Te. ${ }^{[74]}$ The alloy shows phase transition from hexagonal $(2 \mathrm{H})$, monoclinic $(\beta)$ to orthorhombic $(\mathrm{Td})$ phase with in creased $\mathrm{W}$ component, ${ }^{[70,74]}$ which pave the way for phase transition applications. Balicas et al. also proposed that a low content of $\mathrm{W}$ substitution $(\sim 8 \%)$ can stabilize $\gamma$-phase $\mathrm{Mo}_{1-x} \mathrm{~W}_{x} \mathrm{Te}_{2}$ alloy at room temperature ${ }^{[75]}$ The alloy with critical content of $\mathrm{W}$ atoms is sensitive to perturbations such as electric field and strain, which is beneficial for topological phase-transition devices. 


\section{$\mathrm{WS}_{2}$ alloying}

Koratkar et al. proposed that using $\mathrm{WO}_{3}$ and sulfur, adding $\mathrm{NbCl}_{5}$ in the furnace result to p-type $\mathrm{Nb}$ doping during the $\mathrm{WS}_{2} \mathrm{CVD}$ growth procedure (Figure 3). ${ }^{[67]} \mathrm{NbCl}_{5}$ is placed upstream under a heating belt, when the temperature reaches $900{ }^{\circ} \mathrm{C}$. The heating belt is turned on to about $100{ }^{\circ} \mathrm{C}$. The growth procedure is conducted at $80 \mathrm{sccmAr}$ gas and $200 \mathrm{mTorr}$ pressure, and about $6.7 \% \mathrm{Nb}$ atoms replacement is realized. Remarkably, the bandgap is reduced but the direct bandgap nature is preserved. ${ }^{[67]} \mathrm{Nb}$ alloying only changes hole concentration, and the hole mobility is unchanged. Due to the p-type doping nature, the PL changes can be also explained based on exciton and trion changes experimentally and theoretically. Remarkably, for other doped materials, mechanisms for electronic and optoelectronic property changes are still under investigation. Furthermore, n-type Re dopping on $\mathrm{MoS}_{2}$ can be achieved using the same mechanism. ${ }^{[67]}$ $\mathrm{ReO}_{3}$ and $\mathrm{MoO}_{3}$ precursors are placed in two different zones, with growth temperature of 400 and $600{ }^{\circ} \mathrm{C}$ separately. Due to relative large lattice mismatch, the dopping concentration is low, but such alloy can realize Ohmic contacts, which is beneficial for electronic devices ${ }^{[67]}$ Furthermore, it's worth to be emphasized that doping with oxide or halide precursors is applicable to other TMDs for deliberately n-type or p-type doping theoretically. Therefore, realization of doping with other materials and research into the thermodynamic mechanism is needed.

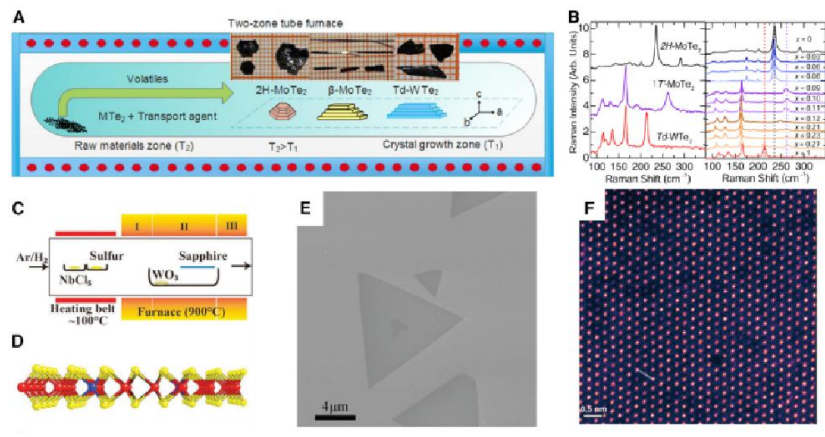

Figure $3 \mathrm{MoTe}_{2}$ and $\mathrm{WS}_{2}$ alloying. (A) Schematic of CVT alloying of $\mathrm{MoTe}_{2}$. (B) Left figure: Raman spectra of $\mathrm{MoTe}_{2}$ with different phases. Right figure: Raman spectra of $\mathrm{Mo}_{1-x} \mathrm{~W}_{x} \mathrm{Te}_{2}$ alloy with different $\mathrm{W}$ contents. The results verify the alloying-induced phase change. (C) Schematic of CVD alloying of $\mathrm{WS}_{2}$. (D) Crystal structure of $\mathrm{Nb}$ dopped $\mathrm{WS}_{2}$. (E) SEM image of Nb-dopped $\mathrm{WS}_{2}$ monolayer triangle islands. (F) Aberration-corrected Z-contrast STEM image of Nb-dopped $\mathrm{WS}_{2}$. (A) is reproduced with permission from Ref. [74]. (B) is reproduced with permission from Ref. [75]. (C-F) are reproduced with permission from Ref. [67].

\section{Quaternary alloying}

Ajayan et al. put forward that increasing the number of components could enhance the possibility to turn band structure of the alloy in a larger scale (Figure 4) ${ }^{[59]}$ Using CVD method to synthesize quaternary $\mathrm{Mo}_{x} \mathrm{~W}_{1-x} \mathrm{~S}_{2 y} \mathrm{Se}_{2(1-y)}$ alloy is achieved by changing the growth temperature, higher temperature leads to uniform distribution of four phases. ${ }^{[59]}$ Growth at lower temperatures $\left(650{ }^{\circ} \mathrm{C}\right)$ only leads to irregular morphology. When the temperature is higher than $700{ }^{\circ} \mathrm{C}$, triangular flakes are grown. ${ }^{[59]}$ For Raman results, the hybrid of in-plane and out-of-plane Raman modes indicates the homogeneous alloying at higher temperature. PL peaks vary from 1.62 to $1.84 \mathrm{eV}$, possessing monotonic peak shift with temperature changes.

Comparing with electrostatic doping or substrate, contamination induced doping, and alloying through CVD methods provides a powerful way to turn electronic properties of TMDs. However, due to atom size differences and constrained alloying conditions, to increase the alloy size for practical applications and reduce defects to enhance

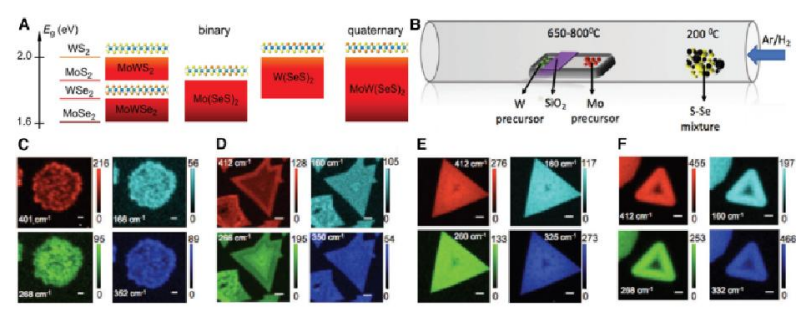

Figure 4 Quaternary $\mathrm{Mo}_{x} \mathrm{~W}_{1-x} \mathrm{~S}_{2 y} \mathrm{Se}_{2(1-y)}$ alloy. (A) Electronic bandgaps of binary alloy and quaternary alloy, which indicates a broader bandgap modulation in quaternary alloy. (B) Schematic of quaternary alloy CVD procedure. (C-F) Raman mapping results for the alloy grown at $650,700,750,800{ }^{\circ} \mathrm{C}$, respectively. The results verify uniform alloy at growth temperature higher than $700{ }^{\circ} \mathrm{C}$. Figure 4 is reproduced with permission from Ref. [59].

device performance are still challenging. Furthermore, alloying with other group-III or group-VI atoms is still needed to be investigated. Most alloys contain only two components. For more complicated alloys with more than two components, efforts are still required.

\section{CVD grown heterostructures}

Due to opposing levels of intrinsic doping of different two-dimensional materials and strong interlayer coupling, two-dimensional materials-based heterostructures are demonstrated for electronic and optoelectronic applications. ${ }^{[13,76-80]}$ While two-dimensional materialsbased vertical heterostructures are fabricated through transfer process generally. However, transfer methods are very complicated for multi-layer structure which lowers success rate. Inevitable rotation misfit has also great influence on device performances. ${ }^{[81]}$ These methods cannot be applied to lateral heterostructures. Meanwhile, contamination imported between layers during transfer can act as dopants or scattering centers which reduces device performances. Furthermore, many materials oxidize quickly in the air, so the transfer process may degrade materials quality. As a result, heterostructures grown through CVD process provide a valid path to solve these challenges. However, alloying is thermodynamically favorable comparing with heterostructure growth generally, so heterostructure growth adopting CVD methods is facing great obstruction in a certain degree. ${ }^{[82]}$

\section{Lateral heterostructure}

The lattice continuality at the grain boundary and confined carrier transport through the lateral junction endow lateral heterostructures with superior in-plane electronic properties. ${ }^{[80,83-85]}$ Therefore, several lateral heterostructures are fabricated through CVD methods by changing chalcogenide atoms or transition metal atoms. Raman, PL and STEM high-angle annular dark-field (HAADF) are common ways to exame heterostructure characters and we'll not discuss them in detail.

Duan et al. have proposed that changing vapor-phase reactants during the growth can result to $\mathrm{WS}_{2}-\mathrm{WSe}_{2}$ lateral heterostructures with relative small lattice mismatch $(\sim 4 \%) .{ }^{[86]}$ However, after the growth of the first material, the edge is easily passivated at ambient conditions so the epitaxial step for the second material is hard. As a result, the authors adopted in situ transfer CVD methods to change reactants in the hot zone of the furnace. At first, the growth of $\mathrm{WS}_{2}$ is carried out at $1057{ }^{\circ} \mathrm{C}$ for 20 min under ambient pressure and a $\mathrm{Ar}$ flow of $70 \mathrm{sccm}$, then the $\mathrm{WS}_{2}$ region is moved out from the hot zone when the furnace is cooled down to $800{ }^{\circ} \mathrm{C}$. At the same time, $\mathrm{WSe}_{2}$ reactants are moved into the hot zone at $1190{ }^{\circ} \mathrm{C}$ under a $\mathrm{Ar}$ flow of $5000 \mathrm{sccm}$ for $20 \mathrm{~min}$. The grown $\mathrm{WS}_{2}-\mathrm{WSe}_{2}$ lateral heterostructures shows excellent rectification behavior for many functional devices. ${ }^{[86]}$ Moreover, the same method can also be applied to $\mathrm{MoS}_{2}-\mathrm{MoSe}_{2}$ lateral heterostructures (Figure $5 .{ }^{[86]}$ 
Ajayan et al. used a one-step CVD method to synthesize $\mathrm{MoS}_{2}$ $\mathrm{WS}_{2}$ heterostructures). ${ }^{[87]}$ Tungsten and tellurium are mixed to grow $\mathrm{WS}_{2}$ while $\mathrm{MoO}_{3}$ and $\mathrm{S}$ powder are placed separately to grow $\mathrm{MoS}_{2}$. Tellurium can help accelerating melting of tungsten, so the one-step growth condition can be achieved. Lower growth temperature at $650{ }^{\circ} \mathrm{C}$ leads to in-plane heterostructure, and higher temperature at $850{ }^{\circ} \mathrm{C}$ leads to vertical heterostructure. We'll refer to the vertical structurelater. Remarkably, the lateral heterostructure edge is along zigzag direction with the seamless connection, and transition between two materials is within a single atomic row. ${ }^{[87]}$ Armchair grain boundary can also be grown, but the edge is not so sharp (1-3 unit cells) due to lower stability. In contrast to sharp edges, the PL peak at the boundary is broadened due to strong intrinsic electric field at the interface which serves as exciton combine center. ${ }^{[87]}$ This phenomenon indicates that the lateral heterostructure can be applied to optoelectronics effectively.

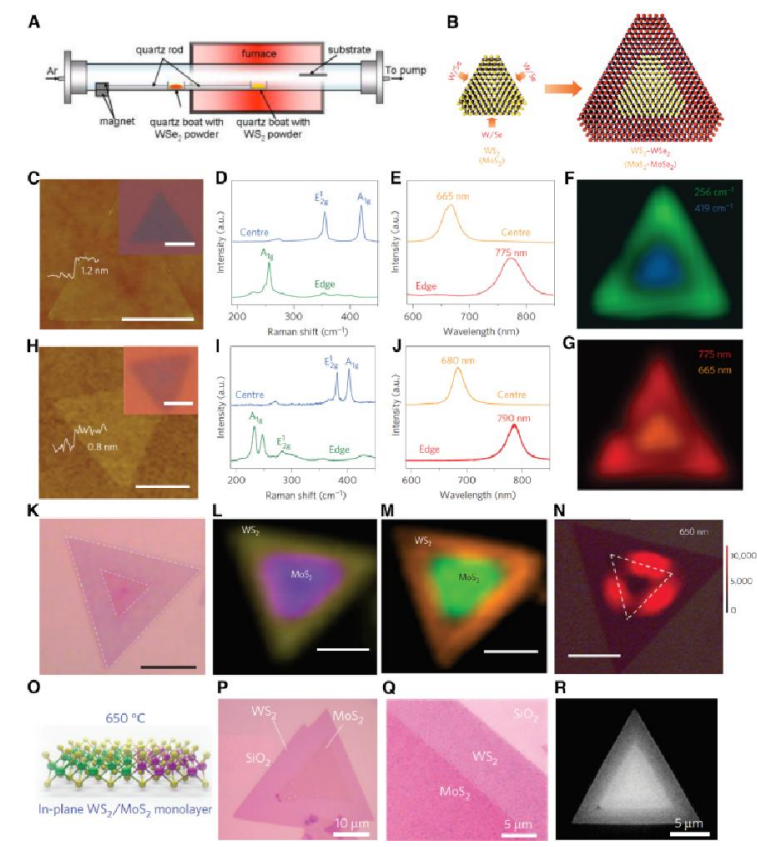

Figure 5 CVD growth of $\mathrm{WS}_{2}-\mathrm{WSe}_{2}, \mathrm{MoS}_{2}-\mathrm{MoSe}_{2}$ and $\mathrm{MoS}_{2}-\mathrm{WS}_{2}$ lateral heterostructures. (A) Schematic of CVD growth of $\mathrm{WS}_{2}-\mathrm{WSe}_{2}$, $\mathrm{MoS}_{2}-\mathrm{MoSe}_{2}$ heterostructures. (B) Crystal structure of $\mathrm{WS}_{2}-\mathrm{WSe}_{2}$, $\mathrm{MoS}_{2}-\mathrm{MoSe}_{2}$ heterostructures. (C) Atomic force microscopy (AFM) mapping of $\mathrm{WS}_{2}-\mathrm{WSe}_{2}$ heterostructure, inset: optical image of $\mathrm{WS}_{2}$ $\mathrm{WSe}_{2}$ heterostructure. Scale bar, $5 \mu \mathrm{m}$. (D-E) Raman and PL spectra of the center and edge part of the $\mathrm{WS}_{2}-\mathrm{WSe}_{2}$ heterostructure. $(\mathrm{F}-\mathrm{G})$ Raman mapping and PL mapping of the $\mathrm{WS}_{2}-\mathrm{WSe}_{2}$ heterostructure. (H) AFM mapping of $\mathrm{MoS}_{2}-\mathrm{MoSe}_{2}$ heterostructure, inset: optical image of $\mathrm{MoS}_{2}-\mathrm{MoSe}_{2}$ heterostructure. Scale bar, $5 \mu \mathrm{m}$. (I-J) Raman mapping and PL mapping of the $\mathrm{MoS}_{2}-\mathrm{MoSe}_{2}$ heterostructure. (K) Optical image of $\mathrm{MoS}_{2}-\mathrm{WS}_{2}$ lateral heterostructure. (L) Combined Raman intensity mapping at $351 \mathrm{~cm}^{-1}$ of $\mathrm{WS}_{2}$ (yellow) and $381 \mathrm{~cm}^{-1}$ of $\mathrm{MoS}_{2}$ (purple). (M) Combined PL intensity mapping at $630 \mathrm{~nm}$ for $\mathrm{WS}_{2}$ (orange) and $680 \mathrm{~nm}$ for $\mathrm{MoS}_{2}$ (green). (N) Strong localized PL enhancement at the interface. The scale bar for $(\mathrm{K}-\mathrm{N}), 10 \mu \mathrm{m}$. (O) Schematic of $\mathrm{WS}_{2}-\mathrm{MoS}_{2}$ lateral heterostructure grown at $650{ }^{\circ} \mathrm{C}$. (P) Optical image of $\mathrm{WS}_{2}-\mathrm{MoS}_{2}$ lateral heterostructure. (Q) Optical image of the interface. (R) SEM image of $\mathrm{WS}_{2}-\mathrm{MoS}_{2}$ lateral heterostructure. (A-J) are reproduced with permission from Ref. [86]. (K-R) are reproduced with permission from Ref. [87].

Besides, Ajayan et al. also proposed a two-step CVD method to control both sizes of the core and shell material for the first time. Their lateral heterostructure can reach $169 \mu \mathrm{m}$, and cross-contamination can be avoided. ${ }^{[88]} \mathrm{MoSe}_{2}$ is grown at $750{ }^{\circ} \mathrm{C}$ for 20 min under
$50 \mathrm{sccm} A r$ flow at first, then the grown $\mathrm{MoSe}_{2}$ is transferred to another furnace to grow $\mathrm{WSe}_{2}$ at $900{ }^{\circ} \mathrm{C}$ under $100 \mathrm{sccmAr}$ flow. Higher temperature can remove small molecules, so passivation at ambient conditions can be ignored, which is slightly different from their work mentioned above. ${ }^{[88]} \mathrm{WSe}_{2}$ can epitaxial grow along $\mathrm{MoSe}_{2}$ edges and on the surface of $\mathrm{MoSe}_{2}$, resulting to both lateral and vertical heterojunction. Interestingly, the second layer $\mathrm{WSe}_{2}$ is firstly grown on edges of $\mathrm{MoSe}_{2}$ instead of common center-originated growth, so prolonged growth time can lead to fully covered $\mathrm{MoSe}_{2}-\mathrm{WSe}_{2}$ vertical heterojunction. Remarkably, high photocurrent response and high efficiency photovoltaic effects expand applications based on $\mathrm{MoSe}_{2}-\mathrm{WSe}_{2}$ lateral heterojunction.

Lee et al. achieved CVD grown $\mathrm{MoS}_{2}-\mathrm{WS}_{2}$ and $\mathrm{MoSe}_{2}-\mathrm{WSe}_{2}$ lateral heterostructures at ambient pressure by applying aromatic molecules as seeding promoters (Figure 6). ${ }^{\left[{ }^{89]}\right.}$ The Mo-based part is grown in lower temperature zone while W-based part is grown in higher temperature zone, the amount of reactants is the key factor. At lower temperatures of $650-700{ }^{\circ} \mathrm{C}$ and longer time, large domain size can be achieved. ${ }^{[89]}$ Under reduced Mo vapor flow, lateral growth is easier to be obtained. Interestingly, both the core material and the shell material of $\mathrm{MoS}_{2}-\mathrm{WS}_{2}$ and $\mathrm{MoSe}_{2}-\mathrm{WSe}_{2}$ lateral heterostructures exhibit 6-fold symmetry second harmonic generation (SHG) intensity with same orientation, which shows that the crystal orientation is on the same direction. ${ }^{[89]}$ No suppression of SHG is observed at the interface, revealing that the heterostructure is of single crystal nature. ${ }^{[89]}$ Meanwhile, the maximum intensity is along 0 degree, which confirms the interface is in zigzag configuration. ${ }^{[89]}$

A vapor transport synthesis method applying $\mathrm{MoO}_{3}$ and $\mathrm{WO}_{3}$ can realize vertical and lateral heterostructure growth, proposed by Jo et $a l .{ }^{[81]} \mathrm{WS}_{2}$ growth at higher temperature is conducted after growth of $\mathrm{MoS}_{2}$ at lower temperature. Under this growth sequence, $\mathrm{WS}_{2}$ is vertically grown above $\mathrm{MoS}_{2}$ edges. ${ }^{[81]}$ However, when the growth sequence is reversed, $\mathrm{MoS}_{2}$ is grown laterally along $\mathrm{WS}_{2}$ edges more favorably. ${ }^{[81]}$ From classical nucleation kinetics model, the free nucleation barrier is relevant to supersaturation, which is determined by temperature difference between crystal melting temperature and growth temperature. ${ }^{[81]}$ Therefore, higher temperature for $\mathrm{WS}_{2}$ growth leads to small supersaturation, yielding vertical epitaxy, while lower temperature for $\mathrm{MoS}_{2}$ possesses high supersaturation on $\mathrm{SiO}_{2}$ surface which leads to lateral growth. ${ }^{[81]}$

$\mathrm{Xu}$ et al. applied physical vapor transport method to grow $\mathrm{MoSe}_{2}-\mathrm{WSe}_{2}$ heterojunctions for only one step. ${ }^{[90]}$ Under $950{ }^{\circ} \mathrm{C}$ with $\mathrm{H}_{2}$ gas flow of $400 \mathrm{sccm}, \mathrm{MoSe}_{2}$ is favorable at first due to rapid evaporation rate. After Mo source is depleted, $\mathrm{WSe}_{2}$ begins to grow. The vapor pressure changes during the whole process are key factor for spontaneous growth of heterojunctions. ${ }^{[90]}$ For one-step CVD methods, such mechanism is also applicable.

It's worth mentioning that most CVD grown heterostructures share the same transition metal atom or chalcogenide atom. However, Li et al. realized lateral $\mathrm{WSe}_{2}-\mathrm{MoS}_{2}$ heterojunction, both transition metal atom and chalcogenide atom are different for the core and shell part. ${ }^{[91]}$ To avoid alloying, $\mathrm{WSe}_{2}$ is grown at higher temperature at first, and $\mathrm{MoS}_{2}$ is grown later. Controlling the relative vapor amount of $\mathrm{MoO}_{3}$ and $\mathrm{S}$ during the $\mathrm{MoS}_{2}$ growth step is essential for sharp interface formation. Excess Mo leads to vertical growth while excess $\mathrm{S}$ promotes $\mathrm{WS}_{2}$ growth. ${ }^{[91]}$ No grain boundary or disorientation is observed in SHG tests. He et al. had grown $\mathrm{WSe}_{2}$ and $\mathrm{MoS}_{2}$ at 925, $755{ }^{\circ} \mathrm{C}$ relatively to form lateral heterojunction. The sharp edge and strong intraface coupling, combined with large active area, enhance photovoltaic carrier generation. ${ }^{[80]}$ The light harvesting efficiency is maintained at a high value of over $95 \%$ even under high angles of incidence from $0^{\circ}$ to $75^{\circ}$, confirming promising applications in solar cells. ${ }^{[80,92]}$ Considering relative large lattice mismatch between these two materials, enlarge crystal size may be difficult. The growth of heterojunctions based on large lattice mismatch materials is still needed to be explored further. 


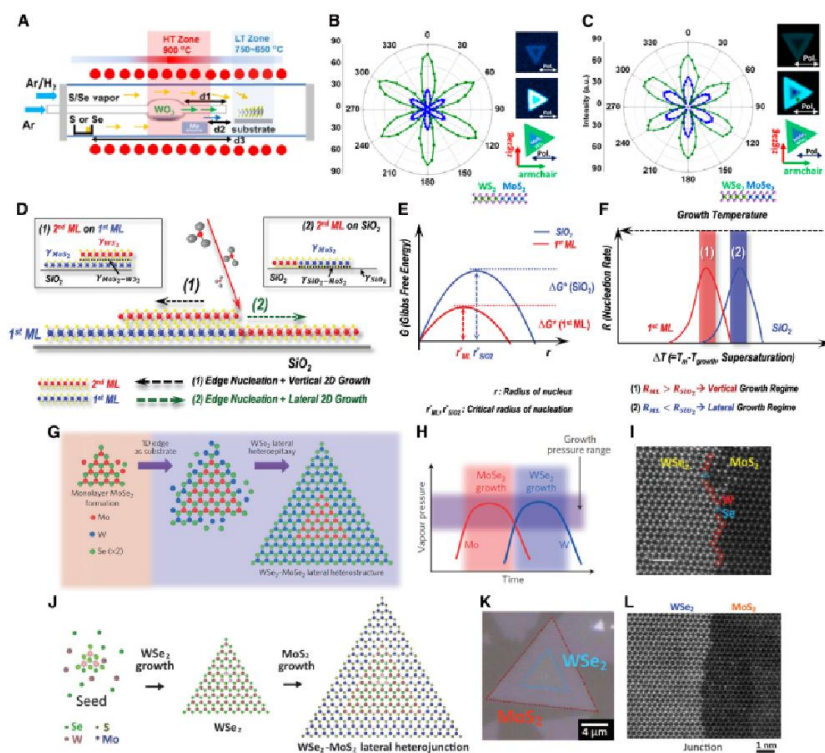

Figure 6 Nucleation mechanism. (A) Schematic of CVD growth of $\mathrm{MoS}_{2}-\mathrm{WS}_{2}$ and $\mathrm{MoSe}_{2}-\mathrm{WSe}_{2}$ lateral heterostructure. (B-C) polarized SHG spectra for $\mathrm{MoS}_{2}-\mathrm{WS}_{2}$ and $\mathrm{MoSe}_{2}-\mathrm{WSe}_{2}$ lateral heterostructur respectively, which confirms the single crystal nature of the heterostructure. (D) Thermodynamic nature for lateral and vertical growth of $\mathrm{WS}_{2}$ and $\mathrm{MoS}_{2}$ based heterostructure. (E) The nucleation energy barrier for the $2^{\text {nd }}$ layer growth on $\mathrm{SiO}_{2}$ and on the $1^{\text {st }}$ monolayer. $(\mathrm{F})$ The relationship between the $2 \mathrm{D}$ nucleation rate and the growth temperature. (G) Growth mechanism for $\mathrm{MoSe}_{2}-\mathrm{WSe}_{2}$ lateral heterojunction. $(\mathrm{H})$ Schematic of vapor pressure changes for the two precursors. (I) STEM image for $\mathrm{MoS}_{2}-\mathrm{WSe}_{2}$ lateral heterostructure with sharp interface. (J) Growth mechanism for $\mathrm{WSe}_{2}-\mathrm{MoS}_{2}$ lateral heterojunction. (K-L) Optical image and STEM image of the grown $\mathrm{WSe}_{2}-$ $\mathrm{MoS}_{2}$ lateral heterojunction. (A-C) are reproduced with permission from Ref. [89]. (D-F) are reproduced with permission from Ref. [81]. $(\mathrm{G}-\mathrm{H})$ are reproduced with permission from Ref. [90]. (I) isreproduced with permission from Ref. [80]. (J-L) are reproduced with permission from Ref. [91].

Gutiérrez et al. changed innovatively gas environment in the presence of water vapor to control the water-induced oxidation and volatilization. As a result, the relative amount of a specific metal oxide vapor can be controlled for lateral epitaxy growth of heterostructures (Figure 7). ${ }^{[93]} \mathrm{N}_{2}$ and $\mathrm{H}_{2} \mathrm{O}$ mixed gas promotes $\mathrm{MoX}_{2}$ growth because only Mo precursors deposited on the substrate, while switching to Ar and $\mathrm{H}_{2}$ mixed gas leads to depleted Mo precursors, W precursors continuously evaporates to the substrate and promotes $\mathrm{WX}_{2}$ growth, where $\mathrm{X}$ represents chalcogenide atom. ${ }^{[93]}$ Both $\mathrm{MoS}_{2^{-}}$ $\mathrm{WS}_{2}$ and $\mathrm{MoSe}_{2}-\mathrm{WSe}_{2}$ heterostructures can be synthesized. The size of the core and shell materials is relevant to growth time and TEM results reveal that the interface is along zigzag directions. ${ }^{[93]} \mathrm{Re}-$ markably, this method can be used to grow multilayer lateral heterostructures with more gas-switching cycles. Furthermore, this gas switching method is also applicable for alloyed heterostructure growth ( $\left.\mathrm{MoS}_{2(1-x)} \mathrm{Se}_{2 x} \mathrm{WS}_{2(1-x)} \mathrm{Se}_{2 x}-\mathrm{MoS}_{2(1-x)} \mathrm{Se}_{2 x}-\mathrm{WS}_{2(1-x)} \mathrm{Se}_{2 x}\right) \quad$ with $\mathrm{MoS}_{2}$ and $\mathrm{WSe}_{2}$ as solid sources, expanding future applications based on this complex structure. ${ }^{[93]}$ Importantly, precursor vapor density is one of key parameters for nucleation and deposition. Introducing gaseous reactants, catalysts or molecular sieves for precursor density control need to be studied further.

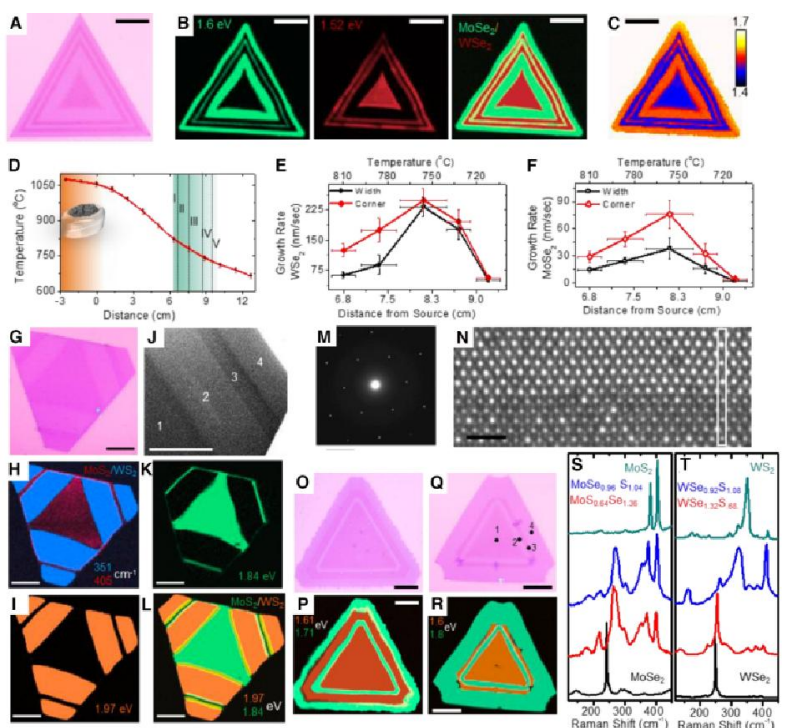

Figure 7 CVD grown multi-junctions. (A) Optical image of $\mathrm{MoSe}_{2}-$ $\mathrm{WSe}_{2}$ multi-junction. (B-C) Corresponding PL peak mapping and PL intensity mapping of $\mathrm{MoSe}_{2}-\mathrm{WSe}_{2}$ multi-junction in (A). (D) Furnace temperature distribution. (E-F) Growth rate for $\mathrm{MoSe}_{2}$ and $\mathrm{WSe}_{2}$ contents in different region marked on (D). (G) Optical image of $\mathrm{MoS}_{2}-\mathrm{WS}_{2}$ multi-junction. (H) Corresponding Raman mapping of $\mathrm{MoS}_{2}-\mathrm{WS}_{2}$ multi-junction on (G). (I-L) PL peak mapping for $\mathrm{MoS}_{2}$, $\mathrm{WS}_{2}$ and the multi-junction on (G). (M) Electron diffraction pattern of the multi-junction. (N) Atomic resolution HAADF-STEMimage of the $\mathrm{MoS}_{2}-\mathrm{WS}_{2}$ interface. (O-P) Multi-junction of $\mathrm{MoS}_{0.64} \mathrm{Se}_{1.36} \mathrm{~W}-$ $\mathrm{S}_{0.68} \mathrm{Se}_{1.32}$ alloys and corresponding PL peak mapping. (Q-R) Multi-junction of $\mathrm{MoS}_{1.04} \mathrm{Se}_{0.96}-\mathrm{WS}_{1.08} \mathrm{Se}_{0.92}$ alloys and corresponding PL peak mapping. (S-T) Raman spectra for $\mathrm{MoS}_{2(1-x)} \mathrm{Se}_{2 x}$ and $\mathrm{WS}_{2(1-x)^{-}}$ $\mathrm{Se}_{2 x}$ alloys on (O) and (Q) respectively. Figure 7 is reproduced with permission from Ref. [93].

While most CVD grown heterostructures show epitaxy nucleation characters where the first grown material forms the core and the next material forms the shell, but Garaj et al. found that temperature dependent in-plane diffusion of sources determines heterostructure growth (Figure 8). ${ }^{[83]}$ They utilized a two-step method to grow $\mathrm{MoS}_{2}-\mathrm{WS}_{2}$ heterojunction, $\mathrm{WS}_{2}$ is grown at first followed by $\mathrm{MoS}_{2}$ growth then. Under $650{ }^{\circ} \mathrm{C}$, a $\mathrm{MoS}_{2}$ core is surrounded by $\mathrm{WS}_{2}$ ring. When the temperature is between $650-710{ }^{\circ} \mathrm{C}$, the whole domain is $\mathrm{Mo}_{1-x} \mathrm{~W}_{x} \mathrm{~S}_{2}$ alloy. The mechanism is that Mo atoms show intra-layer diffusion to substitute $\mathrm{W}$ atoms from the edge to the core, forming the alloying structure, which is sensitive to temperature changes. ${ }^{[83]}$

Xiao et al. used a two-step CVD method to grow GaSe-MoSe heterostructures. ${ }^{[94]} \mathrm{MoSe}_{2}$ is grown through a low-pressure CVD method at first, then GaSe can be laterally or vertically grown along $\mathrm{MoSe}_{2}$ as templates. Lateral heterostructure can be synthesized with larger gas flow. As a result, increased nucleation rate and longer growth time leads to fully covered vertical structure. ${ }^{[94]}$ Due to large lattice mismatch, the lateral heterojunctions at the interface shows an inclination, resulting to elliptical shape and lattice distortion, while vertical heterojunctions possess a supercell of $2.63 \mathrm{~nm} .{ }^{[94]}$ It's noteworthy that the large lattice mismatch will lead to more unique properties of the heterostructure, which are still under investigation.

Duan et al. had an insight into the temperature swing stage between two-step synthesizing methods (Figure 9). ${ }^{[55]}$ The uncontrolled supply of sources always results to undesired homogeneous nuclea- 


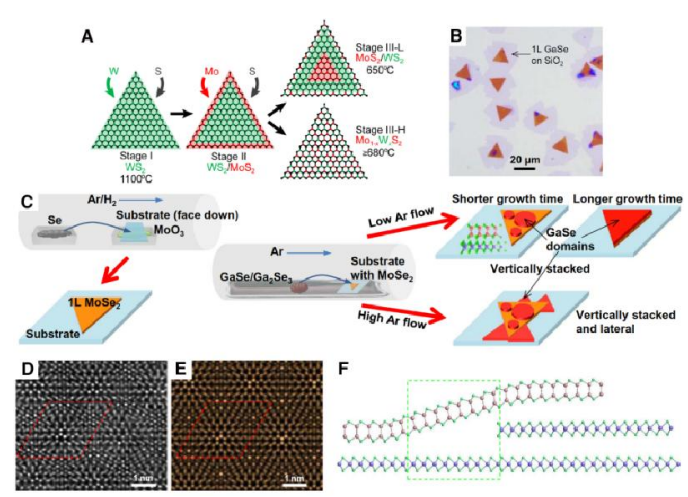

Figure 8 (A) Schematic of diffusion mediated growth of $\mathrm{WS}_{2}$ $\mathrm{MoS}_{2}$ lateral heterostructures. (B) Optical image of GaSe-MoSe vertical and lateral heterostructure. (C) Schematic of growth of GaSe-MoSe $e_{2}$ heterostructures. (D-E) Experimental and simulated results for GaSe-MoSe $e_{2}$ Moiré patterns. (F) Schematic of buckling of $1 \mathrm{~L} \mathrm{GaSe}$ on $\mathrm{MoSe}_{2}$ for the GaSe-MoSe $e_{2}$ lateral heterostructure. (A) is reproduced with permission from Ref. [83]. (B-F) are reproduced with permission from Ref. [94].

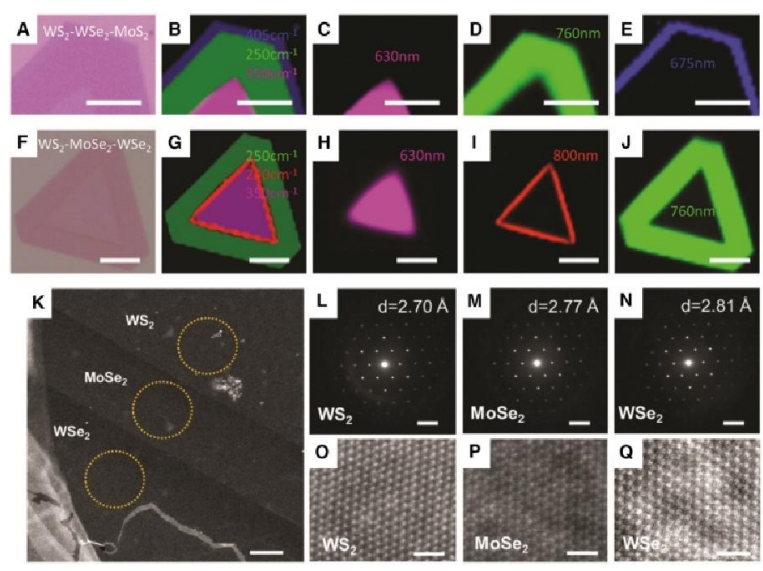

Figure 9 CVD grown heterostructures with multi components. (A) Optical image of $\mathrm{WS}_{2}-\mathrm{WSe}_{2}-\mathrm{MoS}_{2}$ multi-heterostructure. (B) Corresponding Raman mapping on (A). (C-E) Corresponding PL mapping for different parts of the $\mathrm{WS}_{2}-\mathrm{WSe}_{2}-\mathrm{MoS}_{2}$ multi-hetero-structure. (F) Optical image of $\mathrm{WS}_{2}-\mathrm{MoSe}_{2}-\mathrm{WSe}_{2}$ multi-hetero-structure. (G) Corresponding Raman mapping on $(\mathrm{F})$. ( $\mathrm{H}-\mathrm{J})$ Corresponding PL mapping for different parts of the $\mathrm{WS}_{2}-\mathrm{MoSe}_{2}-\mathrm{WSe}_{2}$ multi-heterostructure. (K) Low-magnification Z-contrast image of the $\mathrm{WS}_{2}$ $\mathrm{MoSe}_{2}-\mathrm{WSe}_{2}$ multi-heterostructure. (L-N) Electron diffraction patterns of the different components of $\mathrm{WS}_{2}-\mathrm{MoSe}_{2}-\mathrm{WSe}_{2}$ multi-heterostructure on (K). (O-Q) Atomic-resolution STEM image of the different components of $\mathrm{WS}_{2}-\mathrm{MoSe}_{2}-\mathrm{WSe}_{2}$ multi-heterostructure. Figure 9 is reproduced with permission from Ref. [95].

tion, and the excess thermal degradation hinders realization of sharp interfaces. ${ }^{[95]}$ To control the source supply and thermal degradation effectively, they introduced a reversed gas flow. On the one hand, the grown material at the first stage is cooled down to suppress degradation. On the other hand, unintended supply of chemical vapor source on the target substrate is prevented, so the homogeneous nucleation density is lowered. ${ }^{[95]}$ The authors realized heterostructures and multi-junctions using this method, paving the way for multi-quantumwell researches.

Direct sulfurization or selenization of oxide films is a simple CVD method to realize unigorm CVD films, which is also applicable to in-plane heterostructures. Kim et al. used physical layer deposition (PLD) method to electively deposit $\mathrm{MoO}_{3}$ and $\mathrm{WO}_{3}$ in different re- gion to form $\mathrm{MoSe}_{2}-\mathrm{WSe}_{2}$ heterojunction with sharp interfaces. ${ }^{[76]}$ Such in-plane heterojunctions preserve four Dirac valleys for future valleytronics. ${ }^{[76]}$ However, uniformly deposition of precursors is acquired. Such deposition method is hard to achieve monolayer growth, which hinders its application in some conditions. For example, for devices where direct bandgap or interlayer inversion symmetry is needed.

\section{Vertical heterostructure}

Strong carrier coupling between TMDs gives rise to novel properties, such as ultra-fast carrier transition, ${ }^{[96,97]}$ band alignments, higher energy conversion efficiency, ${ }^{[98]}$ tunneling characters, ${ }^{[99]}$, especially in bilayer heterojunctions. As a result, CVD grown vertical heterostructures has attracted much attention.

Ajayan et al. realized $\mathrm{WS}_{2}-\mathrm{MoS}_{2}$ vertical heterostructure at higher temperatures. Atomic-resolution Z-contrast image shows that the product is uniform bilayer heterostructure with $2 \mathrm{H}$ stacking (Figure 10). ${ }^{[87]}$ Strong interlayer interactions lead to enhanced PL at the step edge region. Owing to the clean interface, more functionalities can be generated from the CVD grown heterostructure with high on-off ratio (larger than $10^{6}$ ) and mobility $\left(15\right.$ to $\left.34 \mathrm{~cm}^{2} \cdot \mathrm{V}^{-1} \cdot \mathrm{s}^{-1}\right){ }^{[87]}$

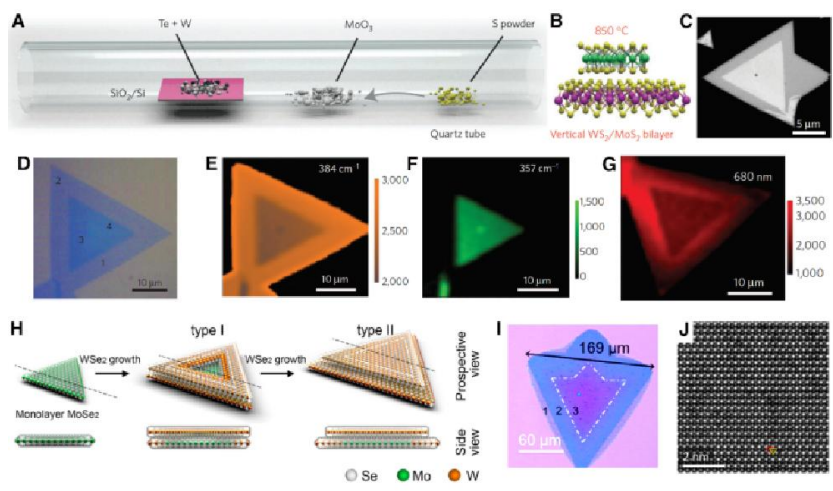

Figure 10 CVD grown $\mathrm{WS}_{2}-\mathrm{MoS}_{2}$ and $\mathrm{WSe}_{2}-\mathrm{MoSe}_{2}$ vertical heterostructure. (A) Schematic of CVD synthesis of $\mathrm{WS}_{2}-\mathrm{MoS}_{2}$ heterostructure. (B) Schematic of the $\mathrm{WS}_{2}-\mathrm{MoS}_{2}$ vertical heterostructure. (C) SEM image of the $\mathrm{WS}_{2}-\mathrm{MoS}_{2}$ vertical heterostructure. (D) Optical image of the $\mathrm{WS}_{2}-\mathrm{MoS}_{2}$ vertical heterostructure. (E-F) Corresponding Raman mapping on (D), the lower intensity of $384 \mathrm{~cm}^{-1}$ on (E) is a result of the coverage of $\mathrm{WS}_{2}$. (G) Enhanced PL intensity at $680 \mathrm{~nm}$ near the step edge of the vertical heterostructure. $(\mathrm{H})$ Schematic of CVD growth of $\mathrm{WSe}_{2}$ above $\mathrm{MoSe}_{2}$ layer. (I) Optical image of the $\mathrm{WSe}_{2}-\mathrm{MoSe}_{2}$ vertical heterostructure. (J) STEM ADF image of the $\mathrm{WSe}_{2}-\mathrm{MoSe}_{2}$ vertical heterostructure. (A-G) are reproduced with permission from Ref. [87]. (H-J) are reproduced with permission from Ref. [88].

Comparing with CVD growth on smooth $\mathrm{SiO}_{2}$ substrate, epitaxial growth on other two-dimensional materials forming vertical heterostructure endows more unique properties. $\mathrm{MoS}_{2}, \mathrm{WS}_{2}$ and $\mathrm{WSe}_{2}$ can be epitaxial grown on $\mathrm{SnS}_{2}$ substrate at relative low temperature (lower than $500{ }^{\circ} \mathrm{C}$ ). ${ }^{[100]}$ The sulfurization of fluorine doped tin oxide (FTO) was achieved at $500-575{ }^{\circ} \mathrm{C}$ for $10 \mathrm{~min}$ at first, resulting in 2T-type $\mathrm{SnS}_{2}$. Then $\mathrm{MoX}_{2}$ can be epitaxial growth on $\mathrm{SnS}_{2}$ (Figure 11). ${ }^{[100]}$ However, the growth temperature of the second material should be carefully controlled to maintain good quality of $\mathrm{SnS}_{2}$ and the grown material. ${ }^{[100]}$ Furthermore, Jin et al. have grown $\mathrm{MoS}_{2}$ on $\mathrm{SnS}_{2}, \mathrm{TaS}_{2}$, and graphene with layer controllability. ${ }^{[101]}$ The use of metal halide precursors at lower temperatures is more efficient for heterostructure growth, consistent with their previous work. ${ }^{[100]} \mathrm{Sul}$ furization of $\mathrm{SnO}_{2}$ at $850{ }^{\circ} \mathrm{C}$ leads to $\mathrm{SnS}_{2}$ layers, then $\mathrm{MoCl}_{5}$ precursor is used to grow $\mathrm{MoS}_{2}$. Since $\mathrm{MoCl}_{5}$ is hydroscopic, carefully control the humidity leads to controlled layer numbers of $\mathrm{MoS}_{2}$, which influences partial pressure of the precursor. ${ }^{[100]}$ When all other conditions are the same, decreased humidity leads to thinner $\mathrm{MoS}_{2}$ 
Importantly, $\mathrm{MoS}_{2}$ on other substrates also enriches applications based on diverse heterostructures.

For traditional heterojunctions, orientation mismatch always reduces carrier transfer efficiency. However, Cao et al. have grown $\mathrm{MoS}_{2}-\mathrm{WS}_{2}$ vertical heterostructure by sulfurization of mixed $\mathrm{MoO}_{3}$ and $\mathrm{WO}_{3}$ powder. ${ }^{[102]}$ They proposed that CVD grown $\mathrm{MoS}_{2}-\mathrm{WS}_{2}$ vertical heterostructure shows high transfer efficiency with negligible influences of stacking mismatch, due to strong electron-phonon coupling between two layers. ${ }^{[102]}$ But it's still needed to be noticed that stacking order may have great influences on device performances, ${ }^{[103]}$ especially for exfoliated samples.

For stretchable applications, Ajayan et al. realized CVD synthesis of $\mathrm{WSe}_{2}$ on $\mathrm{MoSe}_{2}$ surface using a two-step method, the heterostructure shows $3 R$ stacking nature. ${ }^{[104]}$ Similar to sole TMDs material, the Raman spectra and PL characters of CVD grown $\mathrm{MoSe}_{2}-\mathrm{WSe}_{2}$ vertical heterojunction also exhibit strain modulation properties, revealing strong interlayer coupling on vertical heterostructures. ${ }^{[104]}$

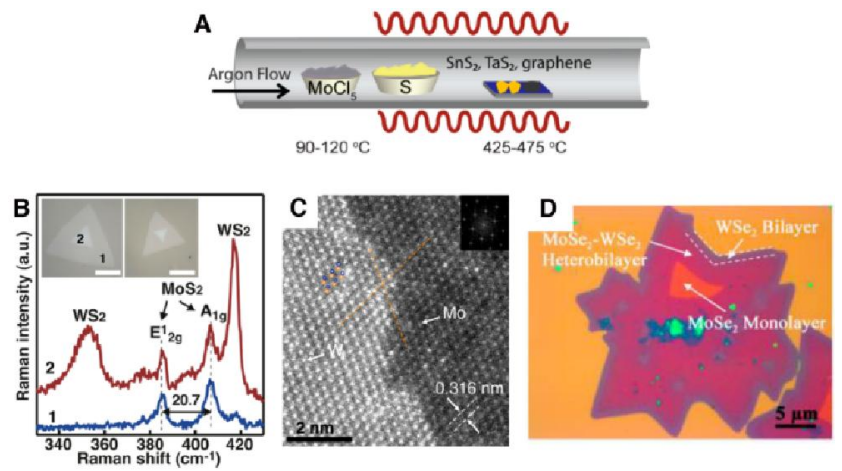

Figure 11 (A) Schematic of epitaxial growth of $\mathrm{MoS}_{2}$ on $\mathrm{SnS}_{2}$. (B) Raman spectra of different areas of the $\mathrm{WS}_{2}-\mathrm{MoS}_{2}$ vertical heterostructure. The inset is the optical image of the heterostructure. Scale bar, $10 \mu \mathrm{m}$. (C) High-angle annular dark field STEM image of the $\mathrm{WS}_{2}-\mathrm{MoS}_{2}$ vertical heterostructure. (D) Optical image of the $\mathrm{MoSe}_{2}-$ $\mathrm{WSe}_{2}$ vertical heterostructure with $3 \mathrm{R}$ stacking. (A) is reproduced with permission from Ref. [100]. (B-C) are reproduced with ermission from Ref. [102]. (D) is reproduced with permission from Ref. [104].

Furthermore, CVD grown heterostructures expand applications for tunneling devices. Robinson et al. used epitaxial multilayer graphene (EG) as the substrate. $\mathrm{WSe}_{2}$ is grown at first implementing a MOCVD method, and then the second layer $\mathrm{MoS}_{2}$ is grown above $\mathrm{WSe}_{2}$ at $750{ }^{\circ} \mathrm{C}$ to form $\mathrm{MoS}_{2}-\mathrm{WSe}_{2}$-EG vertical heterostructure (Figure 12). ${ }^{[105]}$ For $\mathrm{WSe}_{2}-\mathrm{MoSe}_{2}-\mathrm{EG}$ vertical heterostructure, $\mathrm{WSe}_{2}$ layer can be grown on $\mathrm{MoS}_{2}$ layer at $950{ }^{\circ} \mathrm{C}$, and a selenium-sulfur ion exchange would occur at such high temperature for $\mathrm{MoS}_{2}$ layer, tuning $\mathrm{MoS}_{2}$ layer to $\mathrm{MoSe}_{2}$ layer and forming $\mathrm{WSe}_{2}-\mathrm{MoSe}_{2}-\mathrm{EG}$ heterostructures. ${ }^{[105]}$ Remarkably, the graphene substrate with no dangling bond and atomically smooth surface promotes vertical heterostructure growth, where both the transition metal atom and chalcogenide atom are different. ${ }^{[105]}$ Generally, growth on rough surfaces with abundant dangling bonds always results to alloying process. From conductive AFM (CAFM) tunneling current density results, a smaller, resonance tunneling or both phenomenon are easier to occur on $\mathrm{WSe}_{2}-\mathrm{MoSe}_{2}$-EG vertical heterostructures. ${ }^{[105]}$

Generally, heterostructures based on TMDs shows modulated Raman modes and PL peaks. However, Kar et al. found that $\mathrm{MoS}_{2}-\mathrm{Bi}_{2} \mathrm{Se}_{3}$ shows a different electronic band structure. ${ }^{[106]} \mathrm{MoS}_{2}$ is grown by sulfurization of $\mathrm{MoO}_{3}$ at first, then $\mathrm{Bi}_{2} \mathrm{Se}_{3}$ is grown on $\mathrm{MoS}_{2}$ at $480{ }^{\circ} \mathrm{C}$ with $\mathrm{Bi}_{2} \mathrm{Se}_{3}$ powder placed on the hot zone of the furnace. The thickness is controlled by changing deposition time. ${ }^{[106]}$ Such a heterostructure shows novel properties including $100 \%$ pho- toluminescence (PL) quenching, transmittance edge modulation (1.1 to $0.75 \mathrm{eV}$ ), suppressed Raman modes and broad band evolution of spectral transmittance. ${ }^{[106]}$ Attractively, these characters can be reconfigured through a focused laser and the tunable nature of the heterostructure expands future electronic and optoelectronic applications.

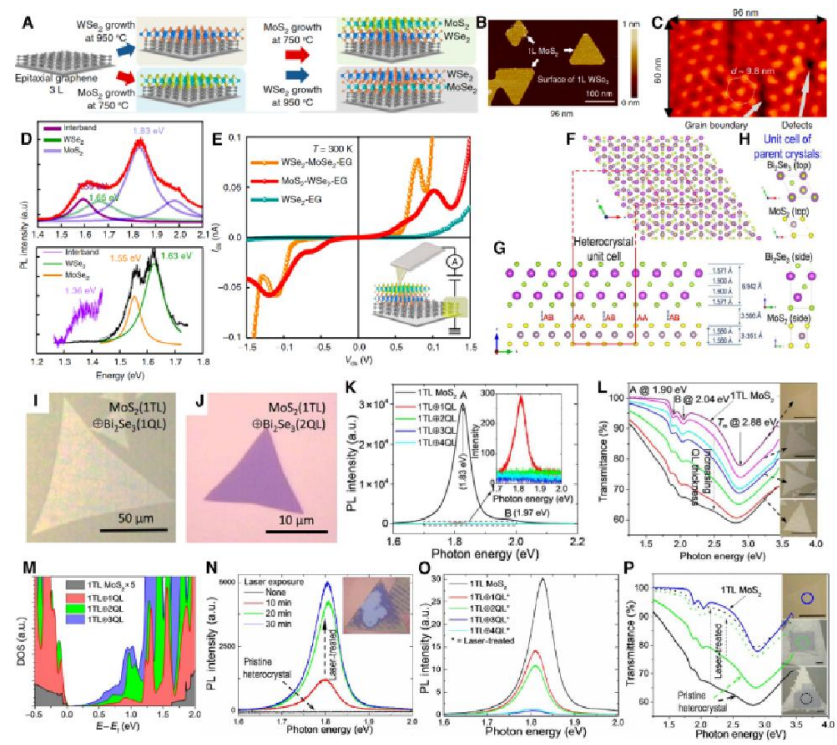

Figure 12 CVD grown heterostructures with novel properties. (A) Schematic of CVD growth of $\mathrm{MoS}_{2}-\mathrm{WSe}_{2}-\mathrm{EG}$ and $\mathrm{WSe}_{2}-\mathrm{MoSe}_{2}-\mathrm{EG}$ vertical heterostructures. (B-C) AFM and STEM image of the Moiré pattern in $\mathrm{MoS}_{2}-\mathrm{WSe}_{2}$. (D) Upper panel: new PL peak at $1.59 \mathrm{eV}$ for $\mathrm{MoS}_{2}$-WSe 2 -EG vertical heterostructure. Lower panel: new PL peak at $1.36 \mathrm{eV}$ for $\mathrm{WSe}_{2}-\mathrm{MoSe}_{2}$-EG vertical heterostructures. Both PL spectra indicates strong interlayer coupling. (E) Resonant tunneling and negative differential resistance characters for $\mathrm{MoS}_{2}-\mathrm{WSe}_{2}-\mathrm{EG}$ and $\mathrm{WSe}_{2}-\mathrm{MoSe}_{2}-\mathrm{EG}$ vertical heterostructures. $(\mathrm{F}-\mathrm{G})$ Crystal geometry for $\mathrm{MoS}_{2}$ trilayers- $\mathrm{Bi}_{2} \mathrm{Se}_{3}$ quintuple layers vertical heterostructure. (H) Top and side view of $\mathrm{MoS}_{2}$ and $\mathrm{Bi}_{2} \mathrm{Se}_{3}$ unit cells. (I-J) Optical image of $\mathrm{MoS}_{2}-\mathrm{Bi}_{2} \mathrm{Se}_{3}$ vertical heterostructure on quartz and $\mathrm{SiO}_{2}$ substrate. (K) Quenched PL peak of $\mathrm{MoS}_{2}$ on $\mathrm{Bi}_{2} \mathrm{Se}_{3}$. (L) Decreased transmittance of $\mathrm{MoS}_{2}$ with increased thickness of $\mathrm{Bi}_{2} \mathrm{Se}_{3}$ quintuple layers. The optical images on the right show increased reflectance of $\mathrm{MoS}_{2}$ flakes. (M) Simulated density of states for $\mathrm{MoS}_{2}-\mathrm{Bi}_{2} \mathrm{Se}_{3}$ vertical heterostructure with increased thickness of $\mathrm{Bi}_{2} \mathrm{Se}_{3}$ quintuple layers. (N) Recovery of PL peak of $\mathrm{MoS}_{2}$ with laser treatment, longer treatment time leads to stronger PL intensity. The inset is the optical image of laser treated heterostructure. (O) Recovery of PL peak of $\mathrm{MoS}_{2}$ for heterostructures with different $\mathrm{Bi}_{2} \mathrm{Se}_{3}$ thickness under laser treatment. (P) Transmittance changes before and after laser treatment. The inset optical images show recovery of pure $\mathrm{MoS}_{2}$ characters after laser treatment. (A-E) are reproduced with permission from Ref. [105]. (F-P) are reproduced with permission from Ref. [106].

Except for combination between TMDs, separately grow TMDs and other layered materials is effective to form heterostructures. $\mathrm{MoS}_{2}$ can be grown on CVD grown h-BN film ${ }^{[107]}$ or graphene ${ }^{[108-113]}$ to form vertical heterostructures. Large area heterostructure film is promising in mass scale integration applications including FET arrays, Hall bar arrays, inverters, tunneling devices, etc. ${ }^{[12]}$ Besides, other method such as molecular beam epitaxy (MBE), combined with CVD, can also realize other van del Waals heterostructures. ${ }^{[114-118]}$ It should be noted that diverse TMDs can form diverse heterostructures, and more novel properties are still needed to be investigated further. The CVD synthesis of heterostructures based on TMDs is just the first step. 


\section{Patterned materials growth}

The strong interlayer interactions between carriers, photons and phonons expands applications of heterostructures, especially in the fields of phototransistors, light-emitting diodes, nanolasers, etc. For multi-functional devices, patterning is drawing much attention from theory ${ }^{[119-121]}$ to real synthesis methods. Currently, many post-growth methods have realized the goal, such as stamp-assisted method, ${ }^{[122,123]}$ electron beam lithography or chemical etching. However, direct growth of pattern is still challenging. Generally, such patterned materials are not grown on flat silicon wafers. To modulate patterned growth, the substrate is needed to be treated, through plasma or chemical treatment to change surface energy, and etched substrate with pore matrix, pyramids or circular cone structures. In this part, we'll briefly introduce several patterned synthesis methods.

For nucleation studies, nucleation of CVD grown flakes or films always starts at substrate edges, scratches, dust particles and rough locations (Figure 13). ${ }^{[105,124]}$ Lou et al. fabricated periodic rectangular $\mathrm{SiO}_{2}$ pillars using lithography method to create substrate edges to control nucleation of $\mathrm{MoS}_{2} .{ }^{[124]}$ Such patterned structure promotes the increase of nucleation density, due to lowered nucleation energy barrier, which is proved in graphene CVD growth. ${ }^{[125]}$ Therefore, the spatial distribution of TMDs flakes can be controlled to form patterns theoretically. However, further researches are needed. Moreover, a deep understanding of nucleation or diffusion mechanism on patterned substrates is still under investigation

Molle et al. had grown $\mathrm{MoS}_{2}$ film on ripple patterned $\mathrm{SiO}_{2} / \mathrm{Si}$ substrate, which is prepared by ion-sputtering. ${ }^{[126]}$ Due to local strain in rippled $\mathrm{MoS}_{2}$, the film shows strong anisotropic Raman and PL spectra, and the anisotropic directions with largest intensity is related to ripple axis. ${ }^{[126]}$ Remarkably, phonon mode shift and localized carrier doping changes, combined with strong exciton binding energy of TMDs pave the way for broad band optoelectronics. ${ }^{[126-128]}$

Except for rippled substrates, patterned substrates with pyramids or circular cone structure are also used to grow TMDs recently. Chueh et al. implemented patterned sapphire substrates to precisely control strain distribution on CVD grown $\mathrm{MoS}_{2}$ films. ${ }^{[129]}$ Due to different thermal expansion coefficients between TMDs and the substrate, the grown material may bear a certain amount of strain. ${ }^{[129,130]}$ The tension strain area can act as quantum well due to narrowed bandgap, while the shrunken strain area with larger bandgap promotes the combination of electrons and holes in the funnel structure ${ }^{[129]}$ Both structures enhance light-matter interactions and absorption of TMDs, which is beneficial for light-harvesting and detection devices. Moreover, topographic design of substrates can also be used to control grain boundary formation during the CVD growth procedure. On the surface of circular cone structure, which processes positive Gaussian curvature, the grown TMDs forms a semi-infinite grain boundary with controlled misfit angel. ${ }^{[131]}$ Combining positive Gaussian curvature structure with negative Gaussian curvature structure, the beginning and ending of grain boundary growth can be precisely controlled. ${ }^{[131]}$ Remarkably, such controlled grain boundary formation gives an insight into thermodynamic nature of defects, and the grown grain boundary shows outstanding properties than those formed on flat substrates, which is promising in carrier scattering control, magnetism, one-dimensional conduction researches, etc. ${ }^{[131-133]}$

Gao et al. have shown that homogeneous monolayer $\mathrm{PtSe}_{2}$ with periodic triangular pattern changing $1 \mathrm{H}$ and $1 \mathrm{~T}$ phases can be grown by controlling the annealing temperature and amount of Se precursors (Figure 14). ${ }^{[134]}$ The selenization of $\mathrm{Pt}$ substrate at $270{ }^{\circ} \mathrm{C}$ is used to grow uniform $1 \mathrm{~T}$ phase $\mathrm{PtSe}_{2},{ }^{[135]}$ but at higher temperature of $400{ }^{\circ} \mathrm{C}$, Se line vacancies with threefold symmetry are energetically preferred, which lead to $1 \mathrm{~T}$ to $1 \mathrm{H}$ phase transform, showing $1 \mathrm{H}$ and $1 \mathrm{~T}$ phase patterns. Reannealing with abundant Se precursors at $270{ }^{\circ} \mathrm{C}$ will restore to uniform $1 \mathrm{~T}$ film. Such patterned $\mathrm{PtSe}_{2}$ is useful to absorb particular molecules forming the same periodic array. In addition, direct selenization of $\mathrm{Cu}$ substrate forms monolayer $\mathrm{CuSe}_{2}$ film with

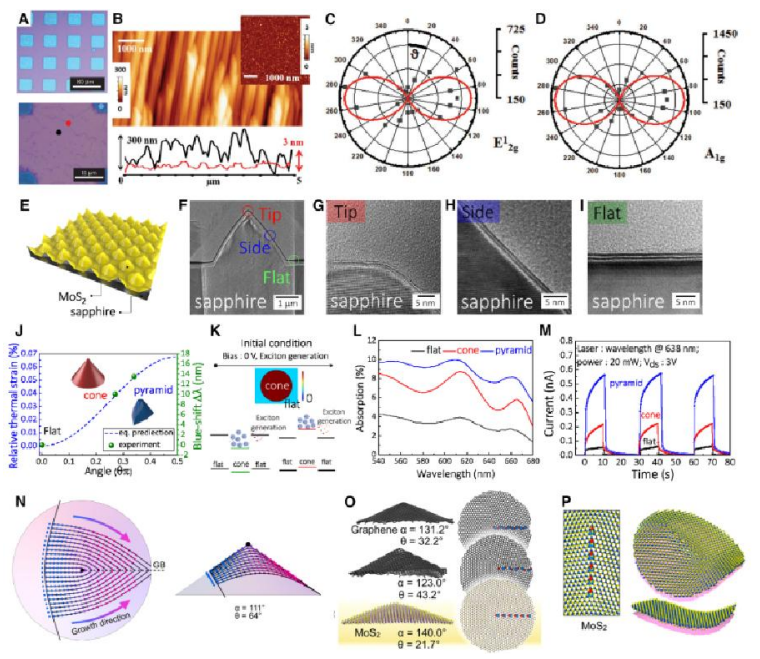

Figure 13 Growth of TMDs on patterned substrates. (A) Upper panel: optical image of $\mathrm{MoS}_{2}$ film on patterned substrate. Lower panel: the close-up optical image. (B) AFM image of rippled $\mathrm{MoS}_{2}$ film and flat $\mathrm{MoS}_{2}$ film (inset). The bottom height profiles show contrast between rippled and flat films. (C-D) Anisotropic $E_{2 \mathrm{~g}}{ }^{1}$ and $\mathrm{A}_{1 \mathrm{~g}}$ Raman peak intensities of the rippled $\mathrm{MoS}_{2}$ film. (E) Schematic of $\mathrm{MoS}_{2}$ film grown on patterned sapphire substrates. (F) TEM image of bilayer $\mathrm{MoS}_{2}$ film grown on patterned sapphire substrates. $(\mathrm{G}-\mathrm{I})$ High-resolution TEM (HRTEM) images from different regions on (F). (J) Theoretical relationship between thermal strain and patterned structure angel. The green points are experimental results. (K) Simulated results for exciton migrated behavior. Type A and B show quantum-well and barrier height behaviors respectively. (L-M) Enhanced absorption and photocurrent for patterned $\mathrm{MoS}_{2}$ film. (N) Schematic of top view (left) and side view (right) of semi-infinite grain boundary grown on the cone structure. $(\mathrm{O})$ Top view and side view of graphene and $\mathrm{MoS}_{2}$ semi-infinite grain boundary with different misfit angles. (P) $\mathrm{MoS}_{2}$ finite grain boundary terminates at local positive and negative Gaussian curvature extrema. (A) is reproduced with permission from Ref. [124]. (B-D) are reproduced with permission from Ref. [126]. (E-M) are reproduced with permission from Ref. [129]. (N-P) are reproduced with permission from Ref. [131].

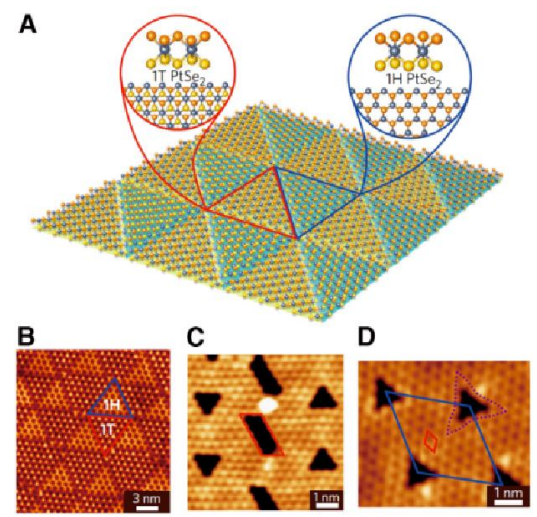

Figure 14 Direct CVD synthesis of patterned TMDs. (A) Schematic of patterned $\mathrm{PtSe}_{2}$ film with alternative $1 \mathrm{~T}$ and $1 \mathrm{H}$ phase triangles. (B) STM image of patterned $\mathrm{PtSe}_{2}$ film. (C) STM image of a domain boundary region of CuSe monolayer. (D) STM image of CuSe film with periodic nanopores. Figure 14 is reproduced with permission from Ref. [134]. 
periodic 13 -atoms-nanopore array. ${ }^{[134]}$ On the synthesis process, to release the lattice mismatch-induced strain, the formation of periodic nanopores is thermodynamically more favorable. On those pores, other molecules can be filled in to produce patterned structure for special functionalities. It's also worth mentioning that the different pattern structure is relevant to the interlayer interactions between the monolayer and the substrate, so more researches into the influence of substrate are needed. ${ }^{[136-138]}$

Researches into patterned TMDs growth is still in the infancy. The patterned structure gives rise to a third-dimension modulation in the two-dimensional plane, so more novel properties are under investigation. Moreover, such patterned structures provide us with a new way to focus on thermodynamic characters of nucleation.

\section{Conclusions and prospects}

Recent years have seen the fast development of TMDs in the fields of electronics, optoelectronics, spin and valley electronics, topological insulators, nonlinear optics, superconductivity, etc. For future practical applications, the challenge is still in the synthesis of large area and high quality TMDs. Currently, most materials cannot be synthesized through CVD methods, so futher studies into novel CVD methods are demanded. From this point of view, thermodynamic nature of TMDs deposition and nucleation is under exploration for single TMDs growth, except for temperature, gas flow rate, furnace pressure, growth time. Other parameters such as substrate treatment, catalysts, precursor preparation are still needed to be considered, especially for the unnoticed changes during the growth procedure. Furthermore, the phase diagram is rather important for TMDS growth, but absence of significant progress reveals that more efforts should be made. For the matured CVD grown TMDs, comparing with exfoliated samples, CVD grown TMDs possessing grain boundary, defects, dust contamination or undesired nucleation center also limits device performances. As a result, for those materials, more efforts have been attracted to researches into controllability of grain boundary, defects. We are sure that the grain boundary and defects formation mechanism is one of the hotspot. Besides this, carrier transport and scattering, interactions with phonon and photon near the grain boundary and defects are also needed to be studied for particular applications.

For lateral and vertical heterostructure growth, it should be noticed that most methods focus on $\mathrm{MoS}_{2}, \mathrm{MoSe}_{2}, \mathrm{WS}_{2}, \mathrm{WSe}_{2}$. Combination with graphene, h-BN is also achieved. However, for other TMDs, the CVD growth of heterostructure is still facing great challenges. Combination with $\mathrm{GaX}, \mathrm{SnX}_{2}, \mathrm{X}$-enes and other layered materials to find novel properties is still in the infancy.

In the past two years, controlled growth of TMDs on patterned substrate has attracted much attention. Due to more complicated structure than the heterostructure, patterned TMDs are more promising to achieve multi-functionalities. Spatial distribution of carrier, phonon, photon interactions, integration with photonic crystals or plasmon matrices may trigger more novel applications. The existence of grain boundary provides us with a new method to control synthesized crystal structure.

In short, the controlled CVD growth of TMDs and precisely tailoring TMDs properties during the CVD procedure are of great significance for the next few years in the field of TMDs researches.

\section{Acknowledgement}

The work was supported by the National Natural Science Foundation of China (No. 61704061).

\section{References}

[1] Xu, M. S.; Liang, T.; Shi, M. M.; Chen, H. Z. Chem. Rev. 2013, 113,
3766.

[2] Splendiani, A.; Sun, L.; Zhang, Y. B.; Li, T. S.; Kim, J.; Chim, C. Y.; Galli, G.; Wang, F. Nano Lett. 2010, 10, 1271.

[3] Sánchez-Pérez, J. R.; Boztug, C.; Chen, F.; Sudradjat, F. F.; Paskiewicz, D. M.; Jacobson, R. B.; Lagally, M. G.; Paiella, R. Proc. Natl. Acad. Sci. 2011, 108, 18893.

[4] Yun, W. S.; Han, S. W.; Hong, S. C.; Kim, I. G.; Lee, J. D. Phys. Rev $B$ 2012, 85, 033305.

[5] Lee, J.; Huang, J. S.; Sumpter, B. G.; Yoon, M. 2D Mater. 2017, 4, 021016 .

[6] Rao, C. N. R.; Matte, H. S. S. R.; Subrahmanyam, K. S.; Maitra, U. Chem. Sci. 2012, 3, 45.

[7] Zhao, J.; Nam, H.; Ly, T. H.; Yun, S. J.; Kim, S.; Cho, S.; Yang, H.; Lee, Y. H. Small 2016, 13, 1601930.

[8] Ko'smider, K.; Fern'andez-Rossier, J. Phys. Rev. B 2013, 87, 075451

[9] Lee, C. H.; Lee, G. H.; van der Zande, A. M.; Chen, W. C.; Li, Y. L.; Han, M. Y.; Cui, X.; Arefe, G.; Nuckolls, C.; Heinz, T. F.; Guo, J.; Hone, J.; Kim, P. Nat. Nanotechnol. 2014, 9, 676.

[10] Xu, W. G.; Liu, W. W.; Schmidt, J. F.; Zhao, W. J.; Lu, X.; Raab, T.; Diederichs, C.; Gao, W. B.; Seletskiy, D. V.; Xiong, Q. H. Nature 2016, 541,62 .

[11] Kozawa, D.; Carvalho, A.; Verzhbitskiy, I.; Giustiniano, F.; Miyauchi, Y.; Mouri, S.; Castro Neto, A. H.; Matsuda, K.; Eda, G. Nano Lett. 2016, 16, 4087.

[12] Pan, S. D.; Ceballos, F.; Bellus, M. Z.; Zereshki, P.; Zhao, H. 2D Mater. 2016, 4, 015033

[13] Fang, H.; Battaglia, C.; Carraro, C.; Nemsak, S.; Ozdol, B.; Kang, J. S.; Bechtel, H. A.; Desai, S. B.; Kronast, F.; Unal, A. A.; Conti, G.; Conlon, C.; Palsson, G. K.; Marting, M. C.; Minor, A. M.; Fadley, C. S.; Yablonovitch, E.; Maboudian, R.; Javey, A. Proc. Natl. Acad. Sci. 2014, 111,6198 .

[14] Chang, T. R.; Xu, S. Y.; Chang, G. Q.; Lee, C. C.; Huang, S. M.; Wang, B. K.; Bian, G.; Zheng, H.; Sanchez, D. S.; Belopolski, I.; Alidoust, N.; Neupane, M.; Bansil, A.; Jeng, H. T.; Lin, H.; Hasan, M. Z. Nat. Commun. 2016, 7, 10639.

[15] Withers, F.; Pozo-Zamudio, O. D.; Mishchenko, A.; Rooney, A. P.; Gholinia, A.; Watanabe, K.; Taniguchi, T.; Haigh, S. J.; Geim, A. K.; Tartakovskii, A. I.; Novoselov, K. S. Nat. Mater. 2015, 14, 301.

[16] Mleczko, M. J.; Zhang, C. F., Lee, H. R.; Kuo, H. H.; Magyari-Köpe, B.; Moore, R. G.; Shen, Z. X.; Fisher, I. R.; Nishi, Y.; Pop, E. Sci. $A d v$. 2017, 3, 1700481.

[17] Shen, T.; Penumatcha, A. V.; Appenzeller, J. ACS Nano 2016, 10 4712.

[18] Xiao, D.; Liu, G.-B.; Feng, W.; Xu, X.; Yao, W. Phys. Rev. Lett. 2012 108,196802

[19] Qian, X.; Liu, J.; Fu, L.; Li, J. Science 2014, 346, 1344.

[20] Zhao, C.; Norden, T.; Zhang, P. Y.; Zhao, P. Q.; Cheng, Y. C.; Sun, F.; Parry, J. P.; Taheri, P.; Wang, J. Q.; Yang, Y. H.; Scrace, T.; Kang, K. F.; Yang, S.; Miao, G. X.; Sabirianov, R.; Kioseoglou, G.; Huang, W.; Petrou, A.; Zeng, H. Nat. Nanotechnol. 2017, 12, 757.

[21] Zheng, F. P.; Cai, C. Y.; Ge, S. F.; Zhang, X. F.; Liu, X.; Lu, H.; Zhang, Y. D.; Qiu, J.; Taniguchi, T.; Watanabe, K.; Jia, S.; Qi, J. S.; Chen, J. H.; Sun, D.; Feng, J. Adv. Mater. 2016, $28,4845$.

[22] Yuan, H. T.; Wang, X. Q.; Lian, B.; Zhang, H. J.; Fang, X. F.; Shen, B.; Xu, G.; Xu, Y.; Zhang, S. C.; Hwang, H. Y.; Cui, Y. Nat. Nanotechnol. 2014, 9,851 .

[23] Jiang, J.; Tang, F.; Pan, X. C.; Liu, H. M.; Niu, X. H.; Wang, Y. X.; Xu, D.F.; Yang, H. F.; Xie, B.P.; Song, F.Q.; Dudin, P.; Kim, T.K.; Hoesch, M.; Das, P. K.; Vobornik, I.; Wan, X. G.; Feng, D. L. Phys Rev. Lett. 2015, 115, 166601

[24] Yang, H.; Kim, S.; Chhowalla, M.; Lee, Y. H. Nat. Phys. 2017, 13, 931.

[25] Cao, T.; Wang, G.; Han, W. P.; Ye, H. Q.; Zhu, C. R.; Shi, J. R.; Niu Q.; Tan, P. H.; Wang, E.; Liu, B. L.; Feng, J. Nat. Commun. 2012, 3, 887.

[26] Zeng, H.; Dai, J.; Yao, W.; Xiao, D.; Cui, X. Nat. Nanotechnol. 2012, 7 , 490 
[27] Mak, K. F.; He, K.; Shan, J.; Heinz, T. F. Nat. Nanotechnol. 2012, 7, 494.

[28] Zhang, Q.; Yang, S. A.; Mi, W.; Cheng, Y.; Schwingenschlögl, U. Adv. Mater. 2015, 28, 959.

[29] Kim, J.; Jin, C. H.; Chen, B.; Cai, H.; Zhao, T.; Lee, P. Y.; Kahn, S.; Watanabe, K.; Taniguchi, T.; Tongay, S.; Crommie, M. F.; Wang, F. Sci. Adv. 2017, 3, 1700518.

[30] Qi, Y. P.; Naumov, P. G.; Ali, M. N.; Rajamathi, C. R.; Schnelle, W.; Barkalov, O.; Hanfland, M.; Wu, S. C.; Shekhar, C.; Sun, Y.; Sü, V.; Schmidt, M.; Schwarz, U.; Pippel, E.; Werner, P.; Hillebrand, R.; Förster, T.; Kampert, E.; Parkin, S.; Cava, R. J.; Felser, C.; Yan, B. H.; Medvedev, S. A. Nat. Commun. 2016, 7, 11038.

[31] Pan, X. C.; Chen, X. L.; Liu, H. M.; Feng, Y. Q.; Wei, Z. X.; Zhou, Y. H.; Chi, Z. H.; Pi, L.; Yen, F.; Song, F. Q.; Wan, X. G.; Yang, Z. R.; Wang, B. G.; Wang, G. H.; Zhang, Y. H. Nat. Commun. 2015, 6, 7805.

[32] Kang, D. F.; Zhou, Y. Z.; Yi, W.; Yang, C. L.; Guo, J.; Shi, Y. G.; Zhang, S.; Wang, Z.; Zhang, C.; Jiang, S.; Li, A.; Yang, K.; Wu, Q.; Zhang, G. M.; Sun, L. L.; Zhao, Z. X. Nat. Commun. 2015, 6, 7804.

[33] Kou, L.; Ma, Y.; Sun, Z.; Heine, T.; Chen, C. J. Phys. Chem. Lett. 2017, 8, 1905.

[34] Sun, Q. L.; Dai, Y.; Niu, C. W.; Ma, Y. D.; Wei, W.; Yu, L.; Huang, B. B. 2D Mater. 2017, 4, 025038.

[35] Zhang, H. J.; Liu, C. X.; Qi, X. L.; Dai, X.; Fang, Z.; Zhang, S. C. Nat. Phys. 2009, 5, 438.

[36] Tang, H.; Liang, D.; Qiu, R. L. J.; Gao, X. P. A. ACS Nano 2011, 5, 7510.

[37] Wang, Y. J.; Liu, E. F.; Liu, H. M.; Pan, Y. M.; Zhang, L. Q.; Zeng, J. W.; Fu, Y. J.; Wang, M.; Xu, K.; Huang, Z.; Wang, Z. L.; Lu, H. Z.; Xing, D. Y.; Wang, B. G.; Wan, X. G.; Miao, F. Nat. Commun. 2016, 7 , 13142.

[38] Ali, M. N.; Xiong, J.; Flynn, S.; Tao, J.; Gibson, Q. D.; Schoop, L. M.; Liang, T.; Haldolaarachchige, N.; Hirschberger, M.; Ong, N. P.; Cava, R. J. Nature 2014, 514, 205.

[39] Zhu, Z. W.; Lin, X.; Liu, J.; Fauqué, B.; Tao, Q.; Yang, C. L.; Shi, Y. G.; Behnia, K. Phys. Rev. Lett. 2015, 114, 176601.

[40] Thoutam, L. R.; Wang, Y. L.; Xiao, Z. L.; Das, S.; Luican-Mayer, A.; Divan, R.; Crabtree, G. W.; Kwok, W. K. Phys. Rev. Lett. 2015, 115, 046602.

[41] Wang, L.; Gutiérrez-Lezama, I.; Barreteau, C.; Ubrig, N.; Giannini, E.; Morpurgo, A. F. Nat. Commun. 2015, 6, 8892.

[42] Chandrasekaran, A.; Mishra, A.; Singh, A. K. Nano Lett. 2017, 17, 3290 .

[43] Xia, F. N.; Wang, H.; Xiao, D.; Dubey, M.; Ramasubramaniam, A. Nat. Photon. 2014, 8, 899.

[44] Li ,Y. Z; Zhang, J. X.; Huang, D. D.; Sun, H.; Fan, F.; Feng, J. B.; Wang, Z.; Ning, C. Z. Nat. Nanotechnol. 2017, 12, 987.

[45] Liu, H. Z.; Li, Y. L.; You, Y. S.; Ghimire, S.; Heinz, T. F.; Reis, D. A. Nat. Phys. 2016, 13, 262

[46] Hong, T.; Chamlagain, B.; Hu, S. R.; Weiss, S. M.; Zhou, Z. X.; Xu, Y. Q. ACS Nano 2015, 9, 5357.

[47] Shi, J.; Yu, P.; Liu, F. C.; He, P.; Wang, R.; Qin, L.; Zhou, J. B.; Li, X.; Zhou, J. D.; Sui, X. Y.; Zhang, S.; Zhang, Y. F.; Zhang, Q.; Sum, T. C.; Qiu, X. H.; Liu, Z.; Liu, X. F. Adv. Mater. 2017, 29, 1701486.

[48] Wu, S. F.; Buckley, S.; Schaibley, J. R.; Feng, L. F.; Yan, J. Q.; Mandrus, D. G.; Hatami, F.; Yao, W.; Vučković, J.; Majumdar, A.; Xu, X. D. Nature 2015, 520, 69.

[49] Zhao, Y. D.; Xu, K.; Pan, F.; Zhou, C. J.; Zhou, F. C.; Chai, Y. $A d v$. Funct. Mater. 2017, 27, 1603484.

[50] Dolui, K.; Rungger, I.; Pemmaraju, C. D.; Sanvito, S. Phys. Rev. B 2013, 88,075420

[51] Ramasubramaniam, A.; Naveh, D. Phys. Rev. B 2013, 87, 195201.

[52] Yang, L. M.; Majumdar, K.; Liu, H.; Du, Y. C.; Wu, H.; Hatzistergos, M.; Hung, P. Y.; Tieckelmann, R.; Tsai, W.; Hobbs, C.; Peide, D.; Ye, P. D. Nano Lett. 2014, 14, 6275.

[53] Kutana, A.; Penev, E. S.; Yakobson, B. I. Nanoscale 2014, 6, 5820.

[54] Zhang, M.; Wu, J. X.; Wu, J. X.; Zhu, Y. M.; Dumcenco, D. O.; Hong, J. H.; Mao, N. N.; Deng, S. B.; Chen, Y. F.; Yang, Y. L.; Jin, C. H.;
Chaki, S. H.; Huang, Y. S.; Zhang, J.; Xie, L. M. ACS Nano 2014, 8 , 7130.

[55] Chen, Y. F.; Xi, J. Y.; Dumcenco, D. O.; Liu, Z.; Suenaga, K.; Wang, D.; Shuai, Z. G.; Huang, Y. S.; Xie, L. M. ACS Nano 2013, 7, 4610.

[56] Li, H. L.; Duan, X. D.; Wu, X. P.; Zhuang, X. J.; Zhou, H.; Zhang, Q. L.; Zhu, X. L.; Hu, W.; Ren, P. Y.; Guo, P. F.; Ma, L.; Fan, X. P.; Wang, X. X.; Xu, J. Y.; Pan, A. L.; Duan, X. F. J. Am. Chem. Soc. 2014, 136, 3756.

[57] Feng, Q. L.; Zhu, Y. M.; Hong, J. H.; Zhang, M.; Duan, W. J.; Mao, N. N.; Wu, J. X.; Xu, H.; Dong, F. L.; Lin, F.; Jin, C. H.; Wang, C. M.; Zhang, J.; Xie, L. M. Adv. Mater. 2014, 26, 2648.

[58] Mann, J.; Ma, Q.; Odenthal, P. M.; Isarraraz, M.; Le, D.; Preciado, E.; Barroso, D.; Yamaguchi, K.; von Son Palacio, G.; Nguyen, A.; Tran, T.; Wurch, M.; Nguyen, A.; Klee, V.; Bobek, S.; Sun, D. Z.; Heinz, T. F.; Rahman, T. S.; Kawakami, R.; Bartels, L. Adv. Mater. 2013, 26, 1399.

[59] Susarla, S.; Kutana, A.; Hachtel, J. A.; Kochat, V.; Apte, A.; Vajtai, R.; Idrobo, J. C.; Yakobson, B. I.; Tiwary C. S.; Ajayan, P. M. Adv. Mater. 2017, 29, 1702457.

[60] Kim, I. S.; Sangwan, V. K.; Jariwala, D.; Wood, J. D.; Park, S.; Chen, K. S.; Shi, F. Y.; Ruiz-Zepeda, F.; Ponce, A.; Jose-Yacaman, M.; Dravid, V. P.; Marks, T. J.; Hersam, M. C.; Lauhon, L. J. ACS Nano 2014, $8,10551$.

[61] Liu, B. L.; Köpf, M.; Abbas, A. N.; Wang, X. M.; Guo, Q. S.; Jia, Y. C.; Xia, F. N.; Weihrich, R.; Bachhuber, F.; Pielnhofer, F.; Wang, H.; Dhall, R.; Cronin, S. B.; Ge, M. Y.; Fang, X.; Nilges, T.; Zhou, C. W. Adv. Mater. 2015, 27, 4423 .

[62] Choi, W.; Cho, M. Y.; Konar, A.; Lee, J. H.; Cha, G. B.; Hong, S. C.; Kim, S.; Kim, J.; Jena, D.; Joo, J.; Kim, S. Adv. Mater. 2012, 24, 5832.

[63] Desai, S. B.; Madhvapathy, S. R.; Sachid, A. B.; Llinas, J. P.; Wang, Q. X.; Ahn, G. H.; Pitner, G.; Kim, M. J.; Bokor, J.; Hu, C. M.; Wong, H.-S. P.; Javey, A. Science 2016, 354, 99.

[64] Gong, Y. J.; Liu, Z.; Lupini, A. R.; Shi, G.; Lin, J. H.; Najmaei, S.; Lin, Z.; Elías, A. L.; Berkdemir, A.; You, G.; Terrones, H.; Terrones, M.; Vajtai, R.; Pantelides, S. T.; Pennycook, S. J.; Lou, J.; Zhou, W.; Ajayan, P. M. Nano Lett. 2014, 14, 442.

[65] Ma, Q.; Isarraraz, M.; Wang, C. S.; Preciado, E.; Klee, V.; Bobek, S.; Yamaguchi, K.; Li, E.; Odenthal, P. M.; Nguyen, A.; Barroso, D.; Sun, D. Z.; Palacio, G. v. S.; Gomez, M.; Nguyen, A.; Le, D.; Pawin, G.; Mann, J.; Heinz, T. F.; Rahman, T. S.; Bartels, L. ACS Nano 2014, 8, 4672 .

[66] Dumcenco, D. O.; Chen, K. Y.; Wang, Y. P.; Huang, Y. S.; Tiong, K. K. J. Alloys Compd. 2010, 506, 940.

[67] Gao, J.; Kim, Y. D.; Liang, L. B.; Idrobo, J. C.; Chow, P.; Tan, J. W.; Li, B. C.; Li, L.; Sumpter, B. G.; Lu, T. M.; Meunier, V.; Hone, J.; Koratkar, N. Adv. Mater. 2016, 28, 9735.

[68] Zhang, K. H.; Feng, S. M.; Wang, J. J.; Azcatl, A.; Lu, N.; Addou, R.; Wang, N.; Zhou, C. J.; Lerach, J.; Bojan, V.; Kim, M. J.; Chen, L. Q.; Wallace, R. M.; Terrones, M.; Zhu, J.; Robinson, J. A. Nano Lett. 2015, 15,6586 .

[69] Suh, J.; Park, T. E.; Lin, D. Y.; Fu, D. Y.; Park, J.; Jung, H. J.; Chen, Y. B.; Ko, C.; Jang, C.; Sun, Y. H.; Sinclair, R.; Chang, J.; Tongay, S.; Wu, J. Q. Nano Lett. 2014, 14, 6976.

[70] Zhang, C. X.; Santosh, K. C.; Nie, Y. F.; Liang, C. P.; Vandenberghe, W. G.; Longo, R. C.; Zheng, Y. P.; Kong, F. T.; Hong, S.; Wallace, R. M.; Cho, K. ACS Nano 2016, 10, 7370.

[71] Li, Y.; Duerloo, K.-A. N.; Wauson, K.; Reed, E. J. Nat. Commun. 2016 , 7, 10671 .

[72] Bera, A.; Singh, A.; Muthu, D. V. S.; Waghmare, U. V.; Sood, A. K. J Phys.: Condens. Matter. 2017, 29, 105403.

[73] Duerloo, K.-A. N.; Li, Y.; Reed, E. J. Nat. Commun. 2014, 5, 4214

[74] Lv, Y. Y.; Cao, L.; Li, X.; Zhang, B. B.; Wang, K.; Pang, B.; Ma, L. G.; Lin, D. J.; Yao, S. H.; Zhou, J.; Chen, Y. B.; Dong, S. T.; Liu, W. C.; Lu, M. H.; Chen, Y. L.; Chen, Y. F. Sci. Rep. 2017, 7, 44587.

[75] Rhodes, D.; Chenet, D. A.; Janicek, B. E.; Nyby, C.; Lin, Y.; Jin, W.; Edelberg, D.; Mannebach, E.; Finney, N.; Antony, A.; Schiros, T.; Klarr, T.; Mazzoni, A.; Chin, M.; Chiu, Y.-C.; Zheng, W.; Zhang, Q. R.; Ernst, F.; Dadap, J. I.; Tong, X.; Ma, J.; Lou, R.; Wang, S.; Qian, T.; 
Ding, H.; Osgood, R. M. Jr.; Paley, D. W.; Lindenberg, A. M.; Huang, P. Y.; Pasupathy, A. N.; Dubey, M.; Hone, J.; Balicas, L. Nano Lett. 2017, 17, 1616.

[76] Ullah, F.; Sim, Y.; Le, C. T.; Seong, M. J.; Jang, J. I.; Rhim, S. H.; Khac, B. C. T.; Chung, K. H.; Park, K.; Lee, Y.; Kim, K.; Jeong, H. Y.; Kim, Y. S. ACS Nano 2017, 11, 8822.

[77] Britnell, L.; Ribeiro, R. M.; Eckmann, A.; Jalil, R.; Belle, B. D.; Mishchenko, A.; Kim, Y.-J.; Gorbachev, R. V.; Georgiou, T.; Morozov, S. V.; Grigorenko, A. N.; Geim, A. K.; Casiraghi, C.; Castro, Neto A. H.; Novoselov, K. S. Science 2013, 340, 1311.

[78] Rubel, O. Sci. Rep. 2017, 7, 4316

[79] Rivera, P.; Schaibley, J. R.; Jones, A. M.; Ross, J. S.; Wu, S. F.; Aivazian, G.; Klement, P.; Seyler, K.; Clark, G.; Ghimire, N. J.; Yan, J. Q.; Mandrus, D. G.; Yao, W.; Xu, X. D. Nat. Commun. 2015, 6, 6242.

[80] Tsai, M. L.; Li, M. Y.; Retamal, J. R. D.; Lam, K. T.; Lin, Y. C.; Suenaga, K.; Chen, L. J.; Liang, G.; Li, L. J.; He, J. H. Adv. Mater. 2017, 29, 1701168.

[81] Heo, H.; Sung, J. H.; Jin, G.; Ahn, J. H.; Kim, K.; Lee, M. J.; Cha, S.; Choi, H.; Jo, M. H. Adv. Mater. 2015, 27, 3803.

[82] Su, S. H.; Hsu, Y. T.; Chang, Y. H.; Chiu, M. H.; Hsu, C. L.; Hsu, W. T.; Chang, W. H.; He, J. H.; Li, L. J. Small 2014, 10, 2589.

[83] Bogaert, K.; Liu, S.; Chesin, J.; Titow, D.; Gradečak, S.; Garaj, S. Nano Lett. 2016, 16, 5129 .

[84] Pulki, A.; Yazyev, O. V. Phys. Rev. B 2016, 93, 041419.

[85] Yu, H.; Kutana, A.; Yakobson, B. Nano Lett. 2016, 16, 5032.

[86] Duan, X. D.; Wang, C.; Shaw, J. C.; Cheng, R.; Chen, Y.; Li, H. L.; Wu, X. P.; Tang, Y.; Zhang, Q. L.; Pan, A. L.; Jiang, J. H.; Yu, R. Q.; Huang, Y.; Duan, X. F. Nat. Nanotechnol. 2014, 9, 1024.

[87] Gong, Y. J.; Lin, J. H.; Wang, X. L.; Shi, G.; Lei, S. D.; Lin, Z.; Zou, X. L.; Ye, G. L.; Vajtai, R.; Yakobson, B. I.; Terrones, H.; Terrones, M; Tay, B. K.; Lou, J.; Pantelides, S. T.; Liu, Z.; Zhou, W.; Ajayan, P. M. Nat. Mater. 2014, 13, 1135.

[88] Gong, Y. J.; Lei, S. D.; Ye, G. L.; Li, B.; He, Y. M.; Keyshar, K.; Zhang, X.; Wang, Q. Z.; Lou, J.; Liu, Z.; Vajtai, R.; Zhou, W.; Ajayan, P. M. Nano Lett. 2015, 15, 6135 .

[89] Zhang, X. Q.; Lin, C. H.; Tseng, Y. W.; Huang, K. H.; Lee, Y. H. Nano Lett. 2014, 15, 410 .

[90] Huang, C. M.; Wu, S. F.; Sanchez, A. M.; Peters, J. J. P.; Beanland, R.; Ross, J. S.; Rivera, P.; Yao, W.; Cobden, D. H.; Xu, X. D. Nat. Mater. 2014, 13, 1096.

[91] Li, M. Y.; Shi, Y. M.; Cheng, C. C.; Lu, L. S.; Lin, Y. C.; Tang, H. L.; Tsai, M. L.; Chu, C. W.; Wei, K. H.; He, J. H.; Chang, W. H.; Suenaga, K.; Li, L. J. Science 2015, 349, 524

[92] Ahn, J.; Jeon, P. J.; Raza, S. R. A.; Pezeshki, A.; Min, S. W.; Hwang, D. K.; Im, S. 2D Mater. 2016, 3, 045011

[93] Sahoo, P. K.; Memaran, S.; Xin, Y.; Balicas, L.; Gutiérrez, H. R. Arxiv 2017.

[94] Li, X. F.; Lin, M. W.; Lin, J. H.; Huang, B.; Puretzky, A. A.; Ma, C.; Wang, K.; Zhou, W.; Pantelides, S. T.; Chi, M. F.; Kravchenko, I.; Fowlkes, J.; Rouleau, C. M.; Geohegan, D. B.; Xiao, K. Sci. Adv. 2016, 2, 1501882.

[95] Zhang, Z. W.; Chen, P.; Duan, X. D.; Zang, K. T.; Luo, J.; Duan, X. F. Science 2017, eaan6814.

[96] Hong, X. P.; Kim, J.; Shi, S. F.; Zhang, Y.; Jin, C. H.; Sun, Y. H.; Tongay, S.; Wu, J. Q.; Zhang, Y. F.; Wang F. Nat. Nanotechnol. 2014, 9, 682 .

[97] Tongay, S.; Fan, W.; Kang, J.; Park, J.; Koldemir, U.; Suh, J.; Narang, D. S.; Liu, K.; Ji, J.; Li, J. B.; Sinclair, R.; Wu, J. Q. Nano Lett. 2014, 14,3185 .

[98] Cheng, R.; Li, D. H.; Zhou, H. L.; Wang, C.; Yin, A. X.; Jiang, S.; Liu, Y.; Chen, Y.; Huang, Y.; Duan, X. F. Nano Lett. 2014, 14, 5590.

[99] Li, D.; Chen, M. Y.; Sun, Z. Z.; Yu, P.; Liu, Z.; Ajayan, P. M.; Zhang, Z. X. Nat. Nanotechnol. 2017, 12, 901.

[100] Zhang, X. W.; Meng, F.; Christianson, J. R.; Arroyo-Torres, C.; Lukowski, M. A.; Liang, D.; Schmidt, J. R.; Jin, S. Nano Lett. 2014, 14, 3047 .
[101] Samad, L.; Bladow, S. M.; Ding, Q.; Zhuo, J. Q.; Jacobberger, R. M.; Arnold, M. S.; Jin, S. ACS Nano 2016, 10, 7039.

[102] Yu, Y. F.; Hu, S.; Su, L. Q.; Huang, L. J.; Liu, Y.; Jin, Z. H.; Purezky, A. A.; Geohegan, D. B.; Kim, K. W.; Zhang, Y.; Cao, L. Y. Nano Lett. 2014, 15,486

[103] Hu, X. H.; Kou, L. Z.; Sun, L. T. Sci. Rep. 2016, 6, 31122.

[104] He, Y. M.; Yang, Y.; Zhang, Z. H.; Gong, Y. J.; Zhou, W.; Hu, Z. L.; Ye, G. L.; Zhang, X.; Bianco, E.; Lei, S. D.; Jin, Z. H.; Zou, X. L.; Yang, Y. C.; Zhang, Y.; Xie, E. Q.; Lou, J.; Yakobson, B.; Vajtai, R.; Li, B.; Ajayan, P. Nano Lett. 2016, 16, 3314.

[105] Lin, Y. C.; Ghosh, R. K.; Addou, R.; Lu, N.; Eichfeld, S. M.; Zhu, H.; Li, M. Y.; Peng, X.; Kim, M. J.; Li, L. J.; Wallace, R. M.; Datta, S.; Robinson, J. A. Nat. Commun. 2015, 6, 7311.

[106] Vargas, A.; Liu, F. Z.; Lane, C.; Rubin, D.; Bilgin, I.; Hennighausen, Z.; DeCapua, M.; Bansil, A.; Kar S. Sci. Adv. 2017, 3, 1601741.

[107] Wang, S. S.; Wang, X. C.; Warner, J. H. ACS Nano 2015, 9, 5246.

[108] Miwa, J. A.; Dendzik, M.; Grønborg, S. S.; Bianchi, M.; Lauritsen, J. V.; Hofmann, P.; Ulstrup, S. ACS Nano 2015, 9, 6502.

[109] Chen, C. M.; Feng, Z. H.; Feng, Y. Y.; Yue, Y. C.; Qin, C. Q.; Zhang, D. H.; Feng, W. ACS Appl. Mater. Interfaces 2016, 8, 19004

[110] Dang, W. H.; Peng, H. L.; Li, H.; Wang, P.; Liu, Z. F. Nano Lett. 2010, $10,2870$.

[111] Shi, Y. M.; Zhou, W.; Lu, A. Y.; Fang, W. J.; Lee, Y. H.; Hsu, A. L.; Kim, S. M.; Kim, K. K.; Yang, H. Y.; Li, L. J.; Idrobo, J. C.; Kong, J. Nano Lett. 2012, 12, 2784.

[112] Yu, L. L.; Lee, Y. H.; Ling, X.; Santos, E. J. G.; Shin, Y. C.; Lin, Y. X.; Dubey, M.; Kaxiras, E.; Kong, J.; Wang, H.; Palacios, T. Nano Lett. 2014, 14, 3055.

[113] Lin, Y. C.; Lu, N.; Perea-Lopez, N.; Li, J.; Lin, Z.; Peng, X.; Lee, C. H.; Sun, C.; Calderin, L.; Browning, P. N.; Bresnehan, M. S.; Kim, M. J.; Mayer, T. S.; Terrones, M.; Robinson, J. A. ACS Nano 2014, 8, 3715.

[114] Aretouli, K. E.; Tsipas, P.; Tsoutsou, D.; Marquez-Velasco, J.; Xenogiannopoulou, E.; Giamini, S. A.; Vassalou, E.; Kelaidis, N.; Dimoulas, A. Appl. Phys. Lett. 2015, 106, 143105.

[115] Xenogiannopoulou, E.; Tsipas, P.; Aretouli, K. E.; Tsoutsou, D.; Giamini, S. A.; Bazioti, C.; Dimitrakopulos, G. P.; Komninou, Ph.; Brems, S.; Huyghebaert, C.; Raduc, I. P.; Dimoulas, A. Nanoscale 2015, 7 , 7896.

[116] Diaz, R. C.; Chaghi, R.; Ma, Y. J.; Batzill, M. 2D Mater. 2015, 2, 044010

[117] Aretouli, K. E.; Tsoutsou, D.; Tsipas, P.; Marquez-Velasco, J.; Giamini, S. A.; Kelaidis, N.; Psycharis, V.; Dimoulas, A. ACS Appl. Mater. Interfaces 2016, 8, 23222.

[118] Vishwanath, S.; Liu, X. Y.; Rouvimov, S.; Mende, P. C.; Azcatl, A.; McDonnell, S.; Wallace, R. M.; Feenstra, R. M.; Furdyna, J. K.; Jena, D. 2D Mater. 2015, 2, 024007.

[119] Zhang, H. J.; Li, Y. F.; Hou, J. H.; Tu, K. X.; Chen, Z. F. J. Am. Chem Soc. 2016, 138, 5644

[120] Gao, G. P.; Jiao, Y.; Waclawik, E. R.; Du, A. J. J. Am. Chem. Soc. 2016, 138,6292 .

[121] Zhao, R.; Wang, Y.; Deng, D.; Luo, X.; Lu, W. J.; Sun, Y. P.; Liu, Z. K.; Chen, L. Q.; Robinson, J. Nano Lett. 2017, 17, 3471.

[122] Zhao, J.; Yu, H.; Chen, W.; Yang, R.; Zhu, J. Q.; Liao, M. Z.; Shi, D. X.; Zhang, G. Y. ACS Appl. Mater. Interfaces 2016, 8, 16546.

[123] Hung, Y. H.; Lu, A. Y.; Chang, Y. H.; Huang, J. K.; Chang, J. K.; Li, L. J.; Su, C. Y. ACS Appl. Mater. Interfaces 2016, 8, 20993.

[124] Najmaei, S.; Liu, Z.; Zhou, W.; Zou, X. L.; Shi, G.; Lei, S. D.; Yakobson, B. I.; Idrobo, J. C.; Ajayan, P. M.; Lou, J. Nat. Mater. 2013, 12, 754

[125] Gao, J. F.; Yip, J.; Zhao, J. J.; Yakobson, B. I.; Ding, F. J. Am. Chem. Soc. 2011, 133, 5009 .

[126] Martella, C.; Mennucci, C.; Cinquanta, E.; Lamperti, A.; Cappelluti, E.; de Mongeot, F. B.; Molle, A. Adv. Mater. 2017, 29, 1605785.

[127] Castellanos-Gomez, A.; Roldán, R.; Cappelluti, E.; Buscema, M.; Guinea, F.; van der Zant, H. S. J.; Steele, G. A. Nano Lett. 2013, 13, 5361 . 
[128] Feng, J.; Qian, X. F.; Huang, C. W.; Li, J. Nat. Photon. 2012, 6, 866.

[129] Wang, S. W.; Medina, H.; Hong, K. B.; Wu, C. C.; Qu, Y. D.; Manikandan, A.; Su, T. Y.; Lee, P. T.; Huang, Z. Q.; Wang, Z. M.; Chuang, F. C.; Kuo, H. C.; Chueh, Y. L. ACS Nano 2017, 11, 8768.

[130] Liu, Z.; Amani, M.; Najmaei, S.; Xu, Q.; Zou, X. L.; Zhou, W.; Yu, T.; Qiu, C. Y.; Birdwell, A. G.; Crowne, F. J.; Vajtai, R.; Yakobson, B. I.; Xia, Z. H.; Dubey, M.; Ajayan, P. M.; Lou, J. Nat. Commun. 2014, 5, 5246.

[131] Wang, Y. X.; Crespi, V. H. Nano Lett. 2017, 17, 5297.

[132] Zhang, Z. H.; Zou, X. L.; Crespi, V. H.; Yakobson, B. I. ACS Nano 2013, 7, 10475 .

[133] Liu, H. J.; Jiao, L.; Yang, F.; Cai, Y.; Wu, X. X.; Ho, W.; Gao, C. L.; Jia, J. F.; Wang, N.; Fan, H.; Yao, W.; Xie, M. H. Phys. Rev. Lett. 2014 113, 066105.

[134] Lin, X.; Lu, J. C.; Shao, Y.; Zhang, Y. Y.; Wu, X.; Pan, J. B.; Gao, L.; Zhu, S. Y.; Qian, K.; Zhang, Y. F.; Bao, D. L.; Li, L. F.; Wang, Y. Q.; Liu, Z. L.; Sun, J. T.; Lei, T.; Liu, C.; Wang, J. O.; Ibrahim, K.; Leo- nard, D. N.; Zhou, W.; Guo, H. M.; Wang, Y. L.; Du, S. X.; Pantelides, S. T.; Gao, H. J. Nat. Mater. 2017, 16, 717.

[135] Wang, Y. L.; Li, L. F.; Yao, W.; Song, S. R.; Sun, J. T.; Pan, J. B.; Ren, X.; Li, C.; Okunishi, E.; Wang, Y. Q.; Wang, E. Y.; Shao, Y.; Zhang, Y. Y.; Yang, H. T.; Schwier, E. F.; Iwasawa, H.; Shimada, K.; Taniguchi, M; Cheng, Z. H.; Zhou, S. Y.; Du, S. X.; Pennycook, S. J.; Pantelides, S. T.; Gao, H. J. Nano Lett. 2015, 15, 4013.

[136] Degregorio, Z. P.; Yoo, Y.; Johns, J. E. J. Phys. Chem. Lett. 2017, 8, 1631.

[137] Shi, J. P.; Zhang, X. N.; Ma, D. L.; Zhu, J. B.; Zhang, Y.; Guo, Z. X.; Yao, Y.; Ji, Q. Q.; Song, X. J.; Zhang, Y. S.; Li, C.; Liu, Z. F.; Zhu, W. G.; Zhang, Y. F. ACS Nano 2015, 9, 4017.

[138] Lee, Y. H.; Yu, L. L.; Wang, H.; Fang, W. J.; Ling, X.; Shi, Y. M.; Lin, C. T.; Huang, J. K.; Chang, M. T.; Chang, C. S.; Dresselhaus, M.; Palacios, T.; Li, L. J.; Kong, J. Nano Lett. 2013, 13, 1852. 